\title{
BIRDS
}

\section{6th ANNUAL SASKATCHEWAN CHRISTMAS BIRD COUNT - 1997}

Compiled by WAYNE C. HARRIS Saskatchewan Environment and Resource Management, 350 Cheadle Street West, Swift Current, Saskatchewan. S9H 4G3

The effects of El Niño were a pleasant change after the winter of 1996-97. The fall leading up to the Christmas count period (December 19 - January 4) was unseasonably mild and dry. There was virtually no snow cover throughout the south until just before the New Year and above freezing temperatures during the day were the norm. Water remained open on a number of larger lakes as well as many streams and rivers.

The average overnight low for the entire period was only $-10^{\circ} \mathrm{C}$, the average daytime high $-4^{\circ} \mathrm{C}$ (Table 1 ). If not for the 15 counts carried out after the colder weather arrived on January 1 those averages would have been much higher. The coldest temperatures were reported from Kamsack and Pike Lake with -35 while Eastend - Consul had the warmest at +12 . Overall we went from one of the coldest counts in 1996 to one of the warmest in 1997; will 1998 be average?

A record 103 counts were completed this year, four more than the previous high of 99 in 1991. The total number of participants increased from 666 last year to 857 in 1997, and was 120 more than the former high in 1994. All of this combined to better coverage overall than in any other year. (Table 2).

\section{The Birds}

One hundred and twenty-one species were recorded during the count period, 116 of these on count day with five more seen during the count period but not on count day. In addition to these there were three recognizable subspecies and one colour morph (Table 3, 4 and 5). This far exceeds the 100 species recorded during both 1988 and 1996 counts. Fort Walsh topped the number of species recorded on count day with 43 while Saskatoon's 41 with 4 additional count period species was the best overall. There were four counts which had 40 or more species and seven that had 30 or more.

With a record number of species it is not surprising that the total number of individuals seen is also up. A total of 158,298 individuals were tallied, more than double last year's total and exceeding the previous best year by more than 45,000 . The front runners in most abundant bird category were Canada Goose and Mallard. The 56,832 Canada Geese still in Saskatchewan was almost double the overall number of Canada Geese found in all previous 55 years combined. In fact the 27,800 Canada Geese at Estevan almost equalled the 55 year total! Snow Bunting were surprisingly low on the list ranking $7^{\text {th }}$ in abundance compared to normally $1^{\text {st }}$ or $2^{\text {nd }}$.

\section{New Species}

Three new species were recorded this year bringing the all time list to 167; one on count day and two as count period species. The new count day species was a Western Screech-Owl from Leader South. This bird was found by Brenda and John Flood in a stand of cottonwoods along the South Saskatchewan River. It was very tame and allowed the observers to approach quite close before flushing. Brenda and 
John watched the grey phase owl for more than 15 minutes in excellent light. There is one other possible record of a Western Screech-Owl from the Fort Walsh count in 1984 but this bird was seen in poor light conditions and was thus left as an unidentified ScreechOwl. Western Screech-Owls have also been heard calling in the spring in the Cypress Hills and considering that they nest in the mountains to the west, one could easily follow the Saskatchewan River system to Leader.

The count period species were Hermit Thrush and Orange-crowned Warbler. The Hermit Thrush was found at MacDowall by Myron and Hazel Barton and had been seen several times during the fall but disappeared on December 14. After being absent for more than two weeks it reappeared January 2 with the onset of colder weather and general snow cover. It was feeding on raisins at the feeder and also on elderberries on a nearby shrub. Hermit Thrushes normally winter no farther north than the southern United States except in coastal areas where they are north to British Columbia in winter.

The Orange-crowned Warbler was found in Saskatoon by Ed Driver. The individual was seen on average every $5^{\text {th }}$ day from late fall through to December 31; it was not seen after the onset of cold weather. This individual was usually accompanied by 4 chickadees and 2 nuthatches and preferred to feed in an apple tree. It ate at the apples from near the stem end and also foraged along the branches. Winter distribution of Orange-crowned Warblers is similar to that of the Hermit Thrush, being found in the southern United States and north along the Pacific coast to southern British Columbia; it and the Yellow-rumped are our most hardy warbler species.

\section{Other Rarities}

Table 4 lists the least frequent species on the counts and the rarest species are all in this table. A Lincoln's Sparrow at Raymore was only the fourth record; three of them in the last three years. Savannah Sparrows had only been seen once before but this year were found at both Gardiner Dam and Rockglen - Borderland. For only the second time Ring-necked Duck (Saskatoon), Greater Scaup (Gardiner Dam) and Oldsquaw (Saskatoon) were reported. Single Yellow-rumped Warblers were found at Biggar and Regina providing the $3^{\text {rd }}$ and $4^{\text {th }}$ records.

\section{Population Trends}

Table 7 compares populations of some of the most frequently recorded species. Not surprising given the mild conditions was the tremendous jump in waterfowl numbers. Raptor numbers were relatively stable. With the exception of Ruffed Grouse, grouse numbers were down although the magnitude of grouse declines may have been amplified by the lack of snow which made these species harder to find. Although Evening Grosbeaks were more common than normal, the remainder of the winter finches were much below the normal. Both Snow Bunting and Horned Lark were very scarce but like the grouse the very open conditions may have made them harder to find.

\section{Count Areas and Participants}

(Names of compilers are in italics.)

1.ANGLIN LAKE. Don Buckle, Marshall Gilliland, Mary Gilliland, Debbie Greening, Bessie May Mucho, Suzanne North.

2.ARCHERWILL. Faye Black, Ken Folstad, Pauline Hnetka, Annette Kozak, Judy Revoy, Doris Slind. 
3.ARMIT. Enid Cumming, Anne Harris, Valeri Harris, Wayne Harris, Sheila Lamont, Susan McAdam, Steve Van Wilgenberg.

4.ASSINIBOIA. Ed Bearss, Jack Burgeson, Cecil Hayward, Dave Landa, Delmar Pettem, Wilf Prentice, Ken Schuweiler.

5.BANGOR. Judith Davis, Jean Hilton.

6.BEAUVAL. Leonie Kennedy-Mills, Peter Kennedy-Mills, Susan Leitch, Jim Mills, Clara Sanderson, Rick Sanderson.

7.BIGGAR. Kelly Foster, Guy Wapple, Megan Wapple, Robert Wapple, Sandra Wapple.

8.BIG RIVER. Carla Braidek, Jack Braidek, Joan Braideck.

9.BIRCH HILLS. Marg Mareschal, Moe Mareschal, Don Weidl.

10.BRIGHTWATER RESERVOIR. Keith Hobson, Ed Peters, Alan Smith.

11.BROADVIEW. Marlene Barnes, Ted Barnes, Dave Chaskavich, Barbara Weidl, Don Weidl, Tony Weidl.

12. BROMHEAD. Martin Bailey, Carol Bjorklund.

13.CABRI. Carman Dodge.

14.CHITEK LAKE. Marcel Cornect.

15.CLARK'S CROSSING. Carol Blenkin, Allyson Brady, Nigel Caulkett, Ron Clarke, Darlene Hay, Jim Hay, Marlene Kalanack, Kay Krueger, Gerard Lahey, Garth Nelson, Hilda Noton, Keith Pahl, Bret Parlee, Chad Parlee, Stan Shadick, Martin Stoffel, Hilda Voth, Jim Wedgwood, Colette Wheler, Michael Williams, Jim Wood, Sandra Zdunich.

16.CORONACH. Anne Harris, Valeri Harris, Wayne Harris.

17. CRAVEN. Martin Bailey, Margaret Belcher, Betty Binnie, Carol Bjorklund, Luc Blanchette, Jon Herriot, Kate Herriot, Trevor Herriot, Bob Kreba, Bob Luterbach, Ron Myers, Bill Ogilvie, Karyn Scalise, Kaitlyn Semple, Keiran Semple.
18. CROOKED LAKE. Bill Livsay, Mayta Livsay, Pat Connolley, Boyd Metzler, John Pollock, Dorothy Skene, Ed Skene.

19. CROOKED RIVER. Margaret Mehler, Morley Mehler.

20.CYPRESS HILLS PROVINCIAL PARK (Centre Block). Wayne Harris, Ana Larmour, Brendan Larmour, Debbie Larmour, Don Larmour, Donny Larmour, Marie Larmour, Sean Larmour, Sofia Larmour, Bill MacNab, Jean MacNab, Sue McAdam, Melody Nage/-Hisey.

21.DILKE. Margaret Belcher, Kent Holland, Brian McArton.

22.DUCK LAKE. Keith Hobson, Alan Smith.

23.DUVAL. Enid Cumming, George Herber, Merv Hey, lain Richardson, Lloyd Saul, Steve Van Wilgenberg.

24.EASTEND. Henri Lebastard.

25.EASTEND - CONSUL. Jen Barnes, Evelyn Brown, Robert Gebhardt, Joan Hodgins, Adam Scott, Heidi Scott.

26. EMMA LAKE. GIen Hanson, Jean Hanson, Deanna Krug, Norman Krug.

27.ENDEAVOUR. Norman Harris.

28.ESTEVAN. Anne Harris, Valeri Harris, Wayne Harris, Sheila Lamont.

29.ESTUARY NORTH. Cathy Cocks, Dean Francis.

30.FENTON. Carman Dodge, Dave Stepnisky, Don Weidl.

31.FIFE LAKE. Martin Myers, Robert Rafuse.

32.FORT QU'APPELLE. Elizabeth Aiken, James Armstrong, Phyllis Bordass, Dorothy Chickowski, Errol Cochrane, Anne Davies, Joanne Davies, Tim Davies, Doug Harman, Lillian Hill, Ronald Hooper, Kevin Horseman, Vic Lamontagne, Kaye Lindgren, Jack Lowe, Shirley Lucyk, Don McDougall, Jean McKenna, Allan Mlazgar, Webb Palmer, Paul Paquin, Florence Pearpoint, Lorne 
Rowell, Lloyd Talbot, Gus Vanderpolder, Fred Warren, William Whiting.

33.FORT WALSH. Anne Harris, Valeri Harris, Wayne Harris, Ron Jensen, Burke Korol, Sheila Lamont, Susan McAdam, Wilkes Parsonage, Wayne Renaud, Guy Wapple, Robert Wapple, Jack Wilkinson, Janet Wilkinson.

34.GARDINER DAM. Anne Harris, Valeri Harris, Wayne Harris, Jeff Jensen, Ron Jensen, Burke Korol, Sheila Lamont, Susan McAdam, Wayne Renaud, Guy Wapple, Robert Wapple.

35.GOOD SPIRIT LAKE. Bill Anaka, Joyce Anaka, Julia Wiwchar.

36.GOVENLOCK. Anne Harris, Valeri Harris, Wayne Harris, Ron Jensen, Burke Korol, Sheila Lamont, Sue McAdam, Wayne Renaud, Guy Wapple, Robert Wapple.

37..GRASSLANDS NATIONAL PARK (NW). Pat Fargey, Lynn Grant, Sebastian Yarcoux, Heather Peat, Lori Rissling, Lorie Wiesner, Mike Wynn.

38.GRAYSON. Charles Helm, Daniel Helm, Karl Zimmer.

39.HARRIS. Guy Wapple.

40.HEPBURN. Helen Fehr, Alice Pilatus, Phyllis Siemens.

41. HOLBIEN. Chlorus Harris, Helen Harris.

42. HORSESHOE BEND (North of Kinistino). Daveen Berg, Dannelle Messer, Verna Messer.

43.HUMBOLDT. Ed Brockmeyer, Mike Volk.

44.INDIAN HEAD. Vic Beaulieu, Irv Escott, David Gehl, Roberta Gehl, Ryan Gehl, Gordon Howe, John Kort, Linda Kort, Mary Kort, Allan Nichols, Dora Nichols, Norine Nichols, Laura Poppy, Lorne Scott, Lizza Alarde, Eileen Varley, Gordon Willerth, Garth Willougby, Kathy Willoughby.

45.KAMSACK. John Barisoff, George and Hazel Bernard, Agnes Betz, Mable Buceuk, Marlon Brock, Lindee Dewores,
Bill Koroluik, Mary Lawernce, Laura Loeppky,Adline Nykolishen, Kelsey and Ryley Rezansoff, Isabel Ritchie, Elsie Severson, Elenor Sookocheef, Stan Stone Sr., Evy Sasyniuk, Joyce and Peter Ualow.

46.KELVINGTON. Pat Finnie, Dianne Sloan, Marguerite Sloan.

47. KENASTON. Lawrence Beckie, Margaret Ann Beckie.

48.KENOSEE LAKE. Boyd Metzler, John Pollock.

49.KILWINNING. Alan Daku, Ed Driver, Marg Driver. 50.KINLOCH. Don Forbes, Doreen Forbes, A.J. Greenley, Cliff Logan, Joyce Logan, Sean Reid, Wilf Rodenberg, Ralph Wang, Travis Wang.

51.KUTAWAGAN LAKE. Valeri Harris, Wayne Harris, Sheila Lamont.

52.KYLE. Cliff Matthews, Gordon Moreside.

53.LA RONGE. Jim Paul, Lorie Ann Paul.

54.LAST MOUNTAIN LAKE N.W.A. Valeri Harris, Wayne Harris, Sheila Lamont.

55.LEADER (North). Daisy Meyers.

56.LEADER (South). Brenda Flood, John Flood.

57.LIVELONG. Sarah Pavka.

58.LOVE - TORCH RIVER. Ken Blaine, Lorna Blaine, Bert Dalziel, Duke Dalziel, Joan Dalziel, Kari Dalziel, Nora Dalziel, Sara Dalziel, Anita Deutschmann, Eric Deutschmann, Betty Donovan, Bruce Donovan, Elien L'Heureux, George Lidster, Jean Lidster, Mildred Long, Lynn Matthews, William Matthews.

59.LUSELAND. Kim Finley, Estelle Finley, Graeme Finley, Liam Finley, Bill Frey.

60.MACDOWALL. Myron Barton.

61.MARYFIELD. Daryl Johannesen, Kaitlin Johannesen, Kristine Johannesen. 
62. MAYVIEW. Albert Graham, Judith Graham.

63.MEADOW LAKE. Bill Caldwell, Byron Golly, Stuart Golly, Tyler Golly, Bob Wilson.

64.MELFORT. Phil Curry, Frieda Markland.

65.MELVILLE. Donna Alberts, Bill Barmby, Marion MacLean, Jeanette Olson.

66.MISSINIPE. Shirley Glass.

67.MOOSE JAW. Elsie Adkins, Jack Adkins, Pam Albert, Ron and Vi Anderson, Doug and Helen Brunsdon, Elsie Carrick, Donna and Ron Dommer, Barry and Fern Dowse, Al, David, Terry and Trevor Gurnsey, Kerry and Robert Hanley, Jan Jacobs, Bob and Pat Kern, Eve King, Cy and Leith Knight, Allan Lemieux, Bill McDonald, Connie and Hugh Mclntyre, Jane Anne and Len Mowchenko, Marj Nicolle, Helen and Peter Norys, Gus and Mike Sagal, Arie Van Dorland, Ed and Grace Walker. 68.MOOSE MOUNTAIN. Greg Bobbitt, Ross Douglas, Dick Gutfriend, Keith Sakatch, Stewart Stairmand.

69.NESBIT FOREST WEST (NW of Crutwell). Kim Clark, Kiri Clark, Suzanne Clark, Guy Levesque, Derry Perkin, Don Stenabaugh.

70.NIPAWIN. Carol Blenkin, Vi Budd, Joyce Christiansen, Betty Hodgins, Stefan LeCuyer, Wally Mollburg, Doug Pegg, Helen Pegg, Ruth Thresher.

71.PIKE LAKE. Tony Allen, Melvin Bernbaum, Angie Bernier, Carol Blenkin, Kurt Brauner, Doug and Helen Campbell, Betty Ann and Tom Dunlop, Arnold Ford, Jean Gadd, Mary Gilliland, Bob Girvan, Bernie Gollop, Mike Gollop, Robert Johanson, Logan and Marie Kennedy, Kay Krueger, Gerard Lahey, Joyce Manton, Cliff Matthews, Muriel Miller, Menno Nickel, Hilda Noton, Jack Reid, Frank Roy, Monte Sawyer, Dorothy Schmidt, Doug Schneider, Stan Shadick, Martin Stoffel, George Tosh, Karen Wiebe, Michael Williams, Kay Willson, Phil Willson, Jim Wood, Lois Wooding, John Yuen.
72.PREECEVILLE. Mr. and Mrs. Larry Bartch, Mr. and Mrs. Peter Buryk, Dallas Fairburn, Maureen Johnson, Norman Johnson, Mary Mitchell, Leona Pollock, Mr. and Mrs. William Sawkiw, Muriel Tratch, David Weiman.

73.PRINCE ALBERT. Janice Allan, Les Anderson, Dulcie Barzeele, Murray Batycki, Josh Bosomworth, John Burt, Pamela Burt, Mel Cherwonack, Kim Clark, Carman Dodge, Keith Dodge, Olga Dolan, Charlie French, Jessica French, Jo€ Graumans, Peter Griffiths, Ruth Griffiths, Alice Hambleton, Don Hableton, Albert King, Anne King, Laura King, Colleen Kubica, Lillian Lubky, Don Moriaty, Gerald Murphy, Anne Neely, Don Neely, Derry Perkins, Helen Placsko, Joe Placsko, Bob Reed, Bonnie Reed, Les Senner, Winona Senner, Michael Sheremata, Richard Stevens, Shirley Swain, Joan Thoresen, Jim Town, Brian Tucker, Beryl Wait, Lyn Walker, Don Weidl, Kimberly Weir.

74.PRINCE ALBERT NATIONAL PARK. Susan Carr, Richard Cherepak, Yolanda Cherepak, Bud Dallen, Edith Drury, Michael Fitzsimmons, Dan Frandsen, Ian Friend, Darcy Henderson, Brad Lloyd, Tracy Lloyd, Jill Marlow, Lorrie Marlow, Leanne Martin, Bradley Muir, Pam Nelson, Paula Pasholek, Adam Pidwerbeski, Pauline Smith, Jackie Syroteuk, Marg Tarleton, Michael Tarleton, Paul Tarleton, Peter Tarleton, William Tarleton, Fred Weisberg, Kim Weisberg, Dave Wieder.

75.QU'APPELLE VALLEY DAM. Bob Plaster, Frank Roy, Stan Shadick, Michael Williams.

76.RAYMORE. Anne Harris, Valeri Harris, Wayne Harris, Sheila Lamont.

77.REGINA. Sandy Ayer, Jessie Bailey, Margaret Belcher, Andy Belyk, Lionel Bonneville, Stephane Bonneville, Enid Cumming, Scott Dickin, Bill Dowhaniuk, Robert Ewart, Sandra Ewart, Jonathon Herriot, Kate Herriot, Trevor Herriot, Dale Hjertaas, Estelle Hjertaas, Kerry Hjertaas, Paule Hjertaas, Phyllis Ilsey, Bob Kreba, Carol Kujala, Linda Langenbacher, Real LaRoche, Bob Luterbach, Susan 
McAdam, Anne Marie Murphy, Ron Meyers, Gene Parker, Diether Pescheken, Deeana Poxton, Brian Rainey, Dean Richert, Miriam Richert, Orian Roy-Wright, Karyn Scalise, Frank Switzer, Steve Van Wilgenburg, Teresa Worobey, Robert Wright.

78.ROCKGLEN - BORDERLAND. Martin Myers, Bob Rafuse.

79.ROUND LAKE (Qu'Appelle Valley). Doug Francis, Boyd Metzler, John Pollock, Pat Ward.

80.SALTCOATS. Bill Anaka, Joyce Anaka, Jim Jowsey, Shirley Jowsey, Michael Keenan.

81.SASKATCHEWAN LANDING PROVINCIAL PARK. Calvin Fiala, Val Harris, Wayne Harris, Sig Jordheim, Cameron Lockerbie, Susan McAdam, Dan Zazelenchuk.

82.SASKATCHEWAN RIVER FORKS. Carman Dodge, Beryl Wait, Don Weidl.

83.SASKATOON. Bruce Acton, Darlene Aikman, Nancy Allan, Tony Allen, Juhachi Asai, Kengo Asai, Rika Asai, Sumiko Asai, Howard Baxter, Beverly Beland, Orval Beland, Brian Bentley, Bob Besant, Jeff Besant, Joyce Besant, Eveline Boudreau, Gail Bunt, Leah Currie, Eileen Dalglish, Wes Deptuch, Ed Driver, Marg Driver, Randi Edmonds, Fran Eldridge, Melanie Elliott, Greg Fenty, Cathy Fry, Joe Fry, Martin Gerard, Bernie Gollop, Mike Gollop, Bob Green, Bruce Hanbidge, John Hanbidge, Ramona Harms, Kaija Harris, John Horton, Mary Houston, Stuart Houston, Terri Jackson, Marlene Kalanack, Richard Kerbes, Barbara Kozmyk, Richard Krahn, Kay Kreuger, Gerard Lahey, Erling Larsen, Grethe Larsen, Anna Leighton, Ted Leighton, Louise Roy Mark, Shirley McKercher, Doug McLellan, Cleve mellor, Cathryn Miller, David Miller, Muriel Miller, Allison Muri, Lisa Napier, Garth Nelson, Lillian Nelson, Bruce Noton, Hilda Noton, Keith Pahl, Roy Parker, Bret Parlee, Chad Parlee, Scott Saretzky, Jim Smart, Al Smith, Margo Taylor, Phil Taylor, Ivor Thokle, Orca Thokle, Jeff Turple, Heather Wagg, Jim Wedgwood, George West,
Michael Williams, Jim Wood, Lois Wooding, Dan Zazelenchuk.

\section{SCOTT. Dale Booth, Guy Wapple.}

85.SHAMROCK. Hugh Henry.

86. SHAUNAVON. Mr. and Mrs. Herb. Anderson, Lorri Cowan, Brian Kiss, Carl Osterberg, Marlene Osterberg, Mr. and Mrs. Dick Pirie, Ruth Robertson, Gerry Smith, Fred Wall.

87.SKULL CREEK. Allan Bennetto, Jim Bennetto, Ray Bennetto, Eileen Bennetto, Doris Bircham, Ralph Bircham, Greeba Drever, Bob Nyen, Vivian Nyen, Harvey Wasilow, Kory Wasilow.

88.SNOWDEN. Elenor and Mike Bodnaruk, Irene Hagel, Karen Hagel, Violet Lien, Betty and Harold Pagan, Edna and Percy Pagan, Jack Pickett, Bill and Vera Schemenauer, Bev Smears, Harold and Irene Thompson.

89.SPALDING. Bill Spizawka, Velma Spizawka.

90.SPINNEY HILL. Ed Driver, Alan Smith.

91.SPRUCE HOME. Sharon and Tom Dice.

92.SQUAW RAPIDS. Anne Harris, Valer Harris, Wayne Harris, Sheila Lamont Susan McAdam, Guy Wapple.

93.ST. LUKE (NE of Whitewood). Marleer Barnes, Ted Barnes, Doug Boivin, Caro Bond, Rae Bond, Don Weidl.

94.SWIFT CURRENT. James Beattie, Hugr Henry, Jeff Jensen, Julie Jensen, Ror Jensen, Velma Kinsman, Dorine Kreuger Walt Kreuger, Arlyne Lawson, Douc Lawson, Kim Marsden, Sue McAdam Eloise Miller, Max Miller, Chelse: Stinson, Irene Stinson, Kae Waters, Johr Weston, Pearl Weston, Verdella Zacharias.

95.TISDALE. Carl Mohr, Joyce Mohr.

96.TOGO. Doug Elsasser.

97.TURTLE LAKE. Carol Burand, S. Burand Muriel Carlson, Larry and Enid Eback 
Harold Newton, E.M. Robinson, Vickie Tollefson.

98.WATSON. Doreen MacDonald, Velma Spizawka, Patricia Sterzuk.

99.WELDON. Ada Hawrish, Bernard Hawrish.

100.WEYBURN. Mary Bakken, Leo Belanger, Louise Belanger, Sophie Belanger, Greg Bobbitt, Jim Burge, Bob Cameron, Jeff Cameron, Lucille Cameron, Ros Douglas, Dick Gutfriend, Betty Layh, Phil Layh, Cy Marcotte, Audrey Michel, John Murray, Ray Neville, Keith Sakatch, Norbert Schultz, Stewart Stairmand, Sid Tripoff, Joe Weisgerber.

101.WHITE BEAR. Darryl Jordheim, Floyd Jordheim, sig Jordheim, Greg McCullock, Lynette McCullock.

102.WHITEWOOD. Joe Ashfield, Ellen Blyth, Pat Connalley, Freida Ede, Kelly Finkas, Joyce Jordan, Wilfred Jordens, Bernice Juzyniec, Felix Juzyniec, Jean Meadows, Cindy McKay, Doreen McPhail, IIla Meszanos, Boyd Metzler, Erv Miller, John Pollock, Alfred Pritchard, Florence Pritchard, Larry Reichel, Marj Reichel, Lenore Santo, Diane Veresh, Ernie Veresh, Pat Ward, Edwin Wyatt, Elain Wyatt.

103.YORKTON. Bill Anaka, Joyce Anaka, Dale Hjertaas, Elinor Hjertaas, Kerry Hjertaas, Paule Hjertaas, Warren Hjertaas, Mark Konder, Boyd Metzlar, Ray Riesz, Geoff Rushowick, Patrick Rushowick, Dorothy Skene, Ed Skene, Harold Wilkinson, Wilma Wilkinson, Julia Wiwchar 
Table 1. COUNT WEATHER CONDITIONS (Temperature ${ }^{\circ} \mathrm{C}$, Wind $\mathrm{kmph}$, Snow $\mathrm{cm}$ )

\begin{tabular}{|c|c|c|c|c|c|c|c|c|}
\hline Locality & $\begin{array}{l}\text { Min } \\
T^{\circ} \mathrm{C}\end{array}$ & $\begin{array}{l}\text { Max } \\
\mathrm{T}^{\circ} \mathrm{C}\end{array}$ & $\begin{array}{l}\text { Min } \\
\text { Win }\end{array}$ & $\begin{array}{l}\text { Max } \\
\text { Win }\end{array}$ & $\begin{array}{l}\text { Min } \\
\text { Sno }\end{array}$ & $\begin{array}{l}\text { Max } \\
\text { Sno }\end{array}$ & AM Sky & PM Sky \\
\hline 1. ANGLIN LAKE & -2 & 2 & 0 & 0 & 0 & 3 & partly cloudy & partly cloudy \\
\hline 2. ARCHERWILI & -15 & 0 & 5 & 10 & 10 & 12 & $\begin{array}{l}\text { overcast, m. fog, } \\
\text { light snow }\end{array}$ & $\begin{array}{l}\text { overcast, light fog, } \\
\text { light snow }\end{array}$ \\
\hline 3. ARMIT & -31 & -20 & 5 & 25 & 15 & 20 & clear & clear \\
\hline 4. ASSINIBOIA & -10 & -5 & 5 & 10 & 2 & 5 & partly cloudy & partly cloudy \\
\hline 5. BANGOR & -4 & -2 & 15 & 20 & 0 & 0 & clear & clear \\
\hline 6. BEAUVAL & -30 & -25 & 0 & 0 & 10 & 15 & clear & clear \\
\hline 7. BIGGAR & -10 & 2 & 10 & 40 & 0 & 0 & partly cloudy & mostly clear \\
\hline 8. BIG RIVER & -8 & -2 & 0 & 0 & 0.5 & 2 & mostly clear & mostly clear \\
\hline 9. BIRCH HILLS & -14 & -11 & 6 & 11 & 7 & 10 & overcast, It. snow & overcast \\
\hline 10. BRIGHTWATER & -10 & -5 & 0 & 5 & 0 & 0 & partly cloudy & partly cloudy \\
\hline 11. BROADVIEW & -4 & -1 & 5 & 15 & 0 & 0 & overcast & overcast \\
\hline 12. BROMHEAD & -2 & 0 & 10 & 15 & 0 & 0 & partly cloudy & partly cloudy \\
\hline 13. CABRI & -2 & -1 & 0 & 0 & 0 & 0 & mostly clear & mostly clear \\
\hline 14. CHITEK LAKE & -8 & -2 & 5 & 8 & 0 & 1 & mostly clear & overcast \\
\hline 15. CLARK'S XING & -12 & -1 & 0 & 10 & 0 & 4 & mostly clear & mostly clear \\
\hline 16. CORONACH & -3 & 2 & 10 & 30 & 0 & 0 & clear & clear \\
\hline 17. CRAVEN & -4 & 2 & 5 & 10 & 0 & 0 & mostly clear & mostly clear \\
\hline 18. CROOKED LAKE & -4 & -1 & 10 & 25 & 0 & 0 & partly cloudy & mostly clear \\
\hline 19. CROOKED RIVER & -25 & -23 & 0 & 0 & 15 & 20 & overcast & mostly clear \\
\hline 20. CYPRESS P.P. & 0 & 2 & 0 & 5 & 0 & 10 & clear & mostly clear \\
\hline 21. DILKE & -5 & 5 & 0 & 20 & 0 & 0 & mostly clear & mostly clear \\
\hline 22. DUCK LAKE & -5 & 0 & 0 & 10 & 0 & 5 & overcast & overcast \\
\hline 23. DUVAL & -9 & 0 & 0 & 28 & 0 & 1 & overcast & partly cloudy \\
\hline 24. EASTEND & & 12 & 0 & 5 & 0 & 0 & clear & clear \\
\hline 25. EASTEND & -5 & -3 & $\overline{0}$ & 0 & 0 & 0 & clear & clear \\
\hline 26. EMMA LAKE & -3 & $\begin{array}{ll}0 & \\
\end{array}$ & $\overline{0}$ & 5 & 20 & 25 & overcast; It. snow & overcast \\
\hline 27. ENDEAVOUR & -20 & -5 & 0 & 5 & 0 & 1 & partly cloudy & mostly clear \\
\hline 28. ESTEVAN & -4 & 2 & 10 & 60 & 0 & 0 & overcast & overcast \\
\hline 29. ESTUARY NORTH & -25 & -15 & 5 & 10 & 2 & 3 & partly cloudy & overcast \\
\hline 30. FENTON & -3 & -3 & $\overline{0}$ & 15 & 5 & 10 & overcast, It. snow & overcast, hyy snow \\
\hline 31. FIFE LAKE & -10 & -4 & 5 & 20 & 0 & 0 & mostly clear & mostly clear \\
\hline 32. FT. QU'APPELLE & -15 & -2 & 10 & 15 & 0 & 0 & clear & clear \\
\hline 33. FORT WALSH & -8 & -5 & 10 & 30 & 0 & 1 & overcast & overcast \\
\hline 34. GARDINER DAM & -10 & -5 & 20 & 30 & 0 & 1 & mostly clear & mostly clear \\
\hline 35. GOOD SPIRITL. & -6 & 0 & 0 & 10 & 0 & 1 & overcast & overcast \\
\hline 36. GOVENLOCK & -6 & 2 & $\overline{5}$ & 30 & 0 & 0 & mostly clear & mostly clear \\
\hline 37. GRASSLANDS NP & -20 & -10 & 5 & 10 & 0 & 5 & clear & partly cloudy \\
\hline 38. GRAYSON & -10 & 0 & 0 & 10 & 0 & 1 & mostly clear & mostly clear \\
\hline 39. HARRIS & -7 & 7 & 10 & 60 & 0 & 1 & overcast, lt. rain & partly cloudy \\
\hline 40. HEPBURN & -6 & 0 & & & 0 & 1 & mostly clear & mostly clear \\
\hline 41. HOLBIEN & -6 & -6 & 0 & 0 & 0 & 1 & mostly clear & overcast \\
\hline 42. HORSESHOE B. & -9 & -9 & 0 & 0 & 0 & 0 & overcast & overcast \\
\hline 43. HUMBOLDT & -5 & 2 & 0 & 20 & 0 & 0 & partly cloudy & mostly clear \\
\hline 44. INDIAN HEAD & -25 & -20 & 0 & 5 & 5 & 10 & & mostly clear \\
\hline 45. KAMSACK & -35 & -26 & & & 60 & & clear & \\
\hline 46. KELVINGTON & -11 & -9 & 0 & 0 & 5 & 10 & overcast & overcast \\
\hline 47. KENASTON & -5 & 5 & 10 & 35 & $\overline{0}$ & 1 & overcast & partly cloudy \\
\hline 48. KENOSEE LAKE & -6 & -2 & 0 & 10 & 0 & 2 & partly cloudy & partly cloudy \\
\hline 49. KILWINNING & -10 & -2 & 0 & 5 & 0 & 1 & partly cloudy & partly cloudy \\
\hline 50. KINLOCH & -9 & 0 & 5 & 30 & 0 & 5 & overcast & partly cloudy \\
\hline 51. KUTAWAGAN L. & -5 & 0 & 5 & 15 & 0 & 0 & overcast & overcast \\
\hline 52. KYLE & 2 & 2 & 0 & 0 & 0 & 0 & clear & partly cloudy \\
\hline 53. LARONGE & -6 & -4 & 5 & 15 & 4 & 7 & partly cloudy & overcast \\
\hline
\end{tabular}


Table 1. COUNT WEATHER CONDITIONS (Temperature ${ }^{\circ} \mathrm{C}$, Wind $\mathrm{kmph}$, Snow $\mathrm{cm}$ )

\begin{tabular}{|c|c|c|c|c|c|c|c|c|}
\hline Locality & $\begin{array}{l}\mathrm{Min} \\
\mathrm{T}^{\circ} \mathrm{C}\end{array}$ & $\begin{array}{l}\text { Max } \\
\mathrm{T}^{\circ} \mathrm{C}\end{array}$ & $\begin{array}{l}\text { Min } \\
\text { Win }\end{array}$ & $\begin{array}{l}\text { Max } \\
\text { Win }\end{array}$ & $\begin{array}{l}\text { Min } \\
\text { Sno }\end{array}$ & $\begin{array}{l}\text { Max } \\
\text { Sno }\end{array}$ & AM Sky & PM Sky \\
\hline 54. LAST MT. L. NWA & -10 & 1 & 5 & 25 & 0 & 0] & partly cloudy & clear \\
\hline 55. LEADER (North) & -10 & 4 & 0 & 20 & 0 & 0 & partly cloudy & partly cloudy \\
\hline 56. LEADER (South) & -20 & -18 & 37 & 42 & 2 & 5 & partly cloudy & partly cloudy \\
\hline 57. LVELONG & -15 & -5 & 0 & 0 & 8 & 10 & clear & clear \\
\hline 58. LOVE - TORCHR. & -20 & -10 & 0 & 5 & 10 & 20 & overcast, it. fog & partly cloudy \\
\hline 59. LUSELAND & -8 & -15 & 0 & 15 & 0 & 3 & overcast & partly cloudy \\
\hline 60. MACDOWALL & -24 & -18 & 0 & 2 & 8 & 10 & mostly clear & mostly clear \\
\hline 61. MARYFIELD & -8 & -3 & 5 & 10 & 0 & 0 & partly cloudy & partly cloudy \\
\hline 62. MAWIEW & -8 & -2 & 0 & 0 & 0 & 1 & overcast, It. fog & clear \\
\hline 63. MEADOW LAKE & 0 & 1 & 5 & 10 & 0 & 0 & mostly clear & partly cloudy \\
\hline \multicolumn{9}{|l|}{ 64. MELFORT } \\
\hline 65. MELVILLE & -6 & 1 & 0 & 0 & 0 & 0 & clear & clear \\
\hline 66. MISSINIPE & -17 & -10 & 0 & 5 & 26 & 29 & light fog & moderate fog \\
\hline 67. MOOSE JAW & -4 & 5 & 10 & 19 & 0 & 0 & mostly clear & mostly clear \\
\hline 68. MOOSE MT. & -14 & -3 & 10 & 15 & 0 & 1 & clear & clear \\
\hline 69. NESBIT FOREST & -20 & -10 & 5 & 15 & 6 & 15 & $\begin{array}{l}\text { overcast, } \\
\text { mod. snow }\end{array}$ & $\begin{array}{l}\text { overcast, } \\
\text { mod. snow }\end{array}$ \\
\hline 70. NIPAWIN & -17 & -17 & 0 & 0 & 8 & 8 & clear & mostly clear \\
\hline 71. PIKE LAKE & -35 & -23 & 0 & 10 & 3 & 8 & clear & partly cloudy \\
\hline 72. PREECEVILLE & -1 & 0 & 11 & 13 & 0 & 1 & mostly clear & clear \\
\hline 73. PRINCE ALBERT & -3 & 0 & 15 & 20 & 0 & 0 & Partly cloudy & partly cloudy \\
\hline 74. PR. ALBERT N. P. & -14 & -5 & 5 & 15 & 0 & 4 & mostly clear & partly cloudy \\
\hline 75. QU'APPELLE & 0 & 1 & 15 & 20 & 0 & 1 & partly cloudy & mostly clear \\
\hline 76. RAYMORE & -4 & 3 & 5 & 30 & 0 & 0 & mostly clear & clear \\
\hline 77. REGINA & -13 & 4 & 0 & 10 & 0 & 0 & clear & clear \\
\hline 78. ROCKGLEN & 0 & 6 & 0 & 60 & 0 & 0 & clear & partly cloudy \\
\hline 79. ROUND L. (Q.V.) & -7 & 0 & 0 & 5 & 0 & 0 & mostly clear & partly cloudy \\
\hline 80. SALTCOATS & -7 & -2 & 5 & 10 & 0 & 0 & partly cloudy & overcast \\
\hline 81. SK LANDING PP & -5 & 2 & 10 & 30 & 0 & 0 & overcast & overcast \\
\hline 82. SASK R. FORKS & -15 & -4 & 5 & 10 & 0 & 3 & partly cloudy & mostly clear \\
\hline 83. SASKATOON & -5 & 3 & 0 & 15 & 0 & 2 & mostly clear & partly cloudy \\
\hline 84. SCOTT & -5 & 3 & 10 & 40 & 0 & 1 & overcast & overcast \\
\hline 85. SHAMROCK & -4 & 1 & 15 & 25 & 0 & 0 & partly cloudy & mostly clear \\
\hline 86. SHAUNAVON & -24 & -24 & & & & & clear & clear \\
\hline 87. SKULL CREEK & 4 & 5 & 0 & 0 & 0 & 0 & partly cloudy & partly cloudy \\
\hline 88. SNOWDEN & -12 & 0 & 0 & 0 & 0 & 0.5 & clear & mostly clear \\
\hline 89. SPALDING & -5 & 2 & 0 & 0 & 0 & 5 & mostly clear & mostly clear \\
\hline 90. SPINNEY HILL & -8 & 3 & 5 & 10 & 0 & 2 & overcast, It. fog & partly cloudy \\
\hline 91. SPRUCE HOME & -5 & -4 & 0 & 0 & 7 & 19 & partly cloudy & partly cloudy \\
\hline 92. SQUAW RAPIDS & -25 & -23 & 2 & 15 & 12 & 15 & partly cloudy & partly cloudy \\
\hline 93. ST. LUKE & -2 & 0 & 15 & 15 & 0 & 0.5 & partly cloudy & partly cloudy \\
\hline 94. SWIFT CURRENT & -5 & 2 & 5 & 40 & 0 & 0 & partly cloudy & mostly clear \\
\hline 95. TISDALE & -16 & 0 & 10 & 15 & 0 & 0 & partly cloudy & partly cloudy \\
\hline \multicolumn{9}{|l|}{ 96. TOGO } \\
\hline 97. TURTLE LAKE & -10 & -6 & 0 & 10 & 3 & 5 & clear & partly cloudy \\
\hline 98. WATSON & -26 & -24 & 0 & 0 & 10 & 15 & & mostly clear \\
\hline 99. WELDON & -10 & 0 & 0 & 5 & 0 & 3 & partly cloudy & mostly clear \\
\hline 100. WEYBURN & -8 & -5 & 0 & 10 & 0 & 0 & mostly clear & mostly clear \\
\hline 101. WHITE BEAR & 0 & 3 & 10 & 15 & 0 & 0 & clear & clear \\
\hline 102. WHITEWOOD & -12 & 3 & 0 & 5 & 0 & 0 & mostly clear & mostly clear \\
\hline 103. YORKTON & -20 & -10 & & & 5 & 10 & clear & clear \\
\hline
\end{tabular}




\begin{tabular}{|c|c|c|c|c|c|c|c|c|c|c|c|c|c|c|c|c|c|c|}
\hline \multirow[b]{2}{*}{ LOCATION } & \multirow[b]{2}{*}{ 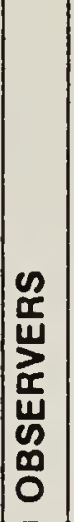 } & \multirow[b]{2}{*}{$\begin{array}{l}5 \\
\text { ட } \\
0 \\
11 \\
z \\
0 \\
z \\
z\end{array}$} & \multirow[b]{2}{*}{$\begin{array}{l}5 \\
0 \\
0 \\
u \\
z \\
0 \\
0 \\
0 \\
x\end{array}$} & \multirow[b]{2}{*}{ 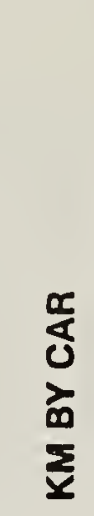 } & \multirow[b]{2}{*}{ 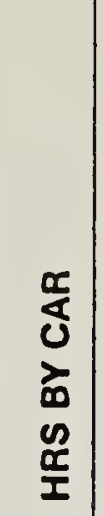 } & \multirow[b]{2}{*}{ 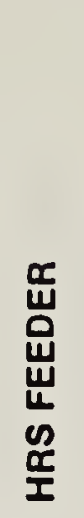 } & \multicolumn{12}{|c|}{$\%$ HABITAT } \\
\hline & & & & & & & 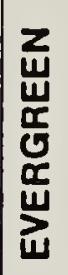 & 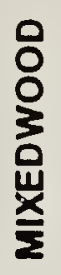 & 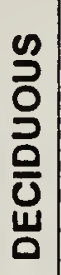 & 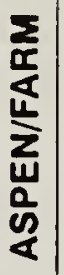 & $\begin{array}{l}\frac{a}{a} \\
\frac{a}{a} \\
\frac{a}{a} \\
\frac{w}{a} \\
\frac{n}{a}\end{array}$ & $\begin{array}{l}\frac{w}{\underline{a}} \\
\frac{\mathbb{a}}{\alpha} \\
\frac{\pi}{\alpha}\end{array}$ & $\begin{array}{l}\frac{5}{o} \\
\frac{\alpha}{\alpha} \\
0 \\
\frac{u}{0} \\
\frac{w}{w} \\
w\end{array}$ & $\begin{array}{l}u \\
0 \\
u \\
\underline{w} \\
5 \\
2 \\
5 \\
5 \\
3\end{array}$ & 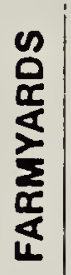 & $\begin{array}{l}z \\
\frac{a}{a} \\
\frac{\infty}{a}\end{array}$ & $\begin{array}{l}\frac{\sigma}{u} \\
u \\
\frac{\alpha}{b} \\
z \\
z \\
u \\
0 \\
0\end{array}$ & $\frac{\frac{\pi}{w}}{\frac{1}{5}}$ \\
\hline 1. ANGLIN LAKE & 6 & 5 & 5 & 0 & 0 & 3 & 15 & 85 & & & & & & & & & & \\
\hline 2. ARCHERWIL & 6 & 3 & 1 & 0 & 0 & 4 & & 10 & 10 & & & & & & 60 & 20 & & \\
\hline 3. ARMIT & 7 & 15 & 7 & 110 & 10 & 0 & 10 & 40 & 40 & 10 & & & & & & & & \\
\hline 4. ASSINIBOIA & 7 & 0 & 0 & 15 & 1 & 0 & & & & & & & & 10 & 80 & 10 & & \\
\hline 5. BANGOR & 2 & 2 & 1 & 20 & 1 & 3 & & & & 10 & 20 & & & 10 & 60 & & & \\
\hline 6. BEAUVAL & 6 & 5 & 7 & 80 & 6 & 0 & 25 & 60 & 10 & & & & 2 & 1 & & 2 & & \\
\hline 7. BIGGAR & 4 & 11 & 5.25 & 202 & 10.25 & 1 & & & & 21 & & & & 29 & 29 & 21 & & \\
\hline 8. BIG RIVER & 3 & 4 & 3 & 0 & 0 & 0.5 & & 70 & & & & & 15 & & 5 & & & $10 a$ \\
\hline 9. BIRCH HILLS & 3 & 4 & 1.25 & 86 & 5 & 2 & & & 5 & 30 & & & 5 & 40 & 10 & 9 & 1 & \\
\hline 10. BRIGHTWATER & 3 & 3 & 2 & 102 & 7.5 & 0 & & & 15 & & 15 & 10 & 5 & 15 & 35 & & 5 & \\
\hline 11. BROADVEW & 6 & 4.5 & 2 & 124 & 5.5 & 0 & 4 & & 40 & 40 & & & & & 4 & 10 & 2 & \\
\hline 12. BROMHEAD & 2 & 0 & 0 & 140 & 6 & 0 & & & & & & & & 95 & 5 & & & \\
\hline 13. CABRI & 1 & 3 & 1 & 110 & 2.75 & 0.5 & & & & & 5 & 5 & & 75 & 5 & 10 & & \\
\hline 14. CHITEK LAKE & 1 & 6 & 3 & 8 & 1.8 & & & 29 & & 18 & & & & 18 & & 30 & & \\
\hline 15. CLARKS XING & 22 & 28.3 & 13.6 & 567 & 31.8 & 0 & & & 6 & 11 & 5 & 1 & 1 & 16 & 26 & 14 & 6 & $5 b, 9 c$ \\
\hline 16. CORONACH & 3 & 2 & 2 & 160 & 5 & & & & & & & 10 & & 60 & 5 & 5 & 20 & \\
\hline 17. CRAVEN & 15 & 20 & 6 & 490 & 22 & & & & & 20 & 10 & 10 & 10 & 30 & 10 & 7 & 3 & \\
\hline 18. CAOOKEDL. & 7 & 2 & 1 & 201 & 8 & 1 & & & 20 & 20 & & & & 10 & 10 & 20 & 20 & \\
\hline 19. CROOKED R. & 2 & 1 & 1 & 25 & 0.75 & 3 & & & & 25 & & & & 25 & 50 & & & \\
\hline 20. CYPRESS PP & 13 & 15 & 4 & 50 & 4 & 3 & 60 & 20 & & & & 10 & & & 10 & & & \\
\hline 21. DILKE & 3 & 0 & 0 & 61 & 1 & 3 & & & & 25 & & & & & 1 & 74 & & \\
\hline 22. DUCK LAKE & 2 & 6 & 3 & 95 & 5.25 & & & 60 & 10 & 15 & 5 & & & & & 10 & & \\
\hline 23. DUVAL & 6 & 7 & 3 & 256 & 10 & 1 & & & 12 & 5 & 5 & 5 & 1 & 35 & 27 & 10 & & \\
\hline 24. EASTEND & 1 & & & & 5 & & 5 & & 40 & & & & & 30 & 5 & 20 & & \\
\hline 25. EASTEND & 6 & & & 150 & 3 & 1 & & & & & & 95 & & 1 & 2 & 2 & & \\
\hline 26. EMMA LAKE & 4 & 2 & 0.5 & 55 & 3 & 3 & & 95 & & & & & & & 5 & & & \\
\hline 27. ENDEAVOUR & 1 & 2 & 2.5 & 30 & 1.5 & 2 & & & 25 & & & & & 20 & 50 & 25 & & \\
\hline 28. ESTEVAN & 4 & 7 & 3 & 150 & 6 & & & & 20 & & & 20 & & 20 & & 20 & 20 & \\
\hline 29. ESTUARYN. & 2 & 1 & 2 & 70 & 3 & 2 & & & 25 & & & 45 & & & 30 & & & \\
\hline 30. FENTON & 3 & 2 & 0.5 & 140 & 4.5 & 1 & & & & 10 & & & 5 & 60 & 15 & 5 & 5 & \\
\hline 31. FIFE LAKE & 2 & 0.5 & 0.5 & 100 & 6 & & & & & & & 5 & & 75 & 5 & & 15 & \\
\hline 32. FT OU'APPEUE & 27 & 3 & 3 & 300 & 10 & 3 & & & 10 & 20 & & 5 & 5 & 30 & 10 & 10 & 10 & \\
\hline 33. FORT WALSH & 13 & 61 & 30.5 & 134 & 28.75 & & & 53 & & & & 1 & & 7 & 7 & & & $32 c$ \\
\hline 34. GARDINER DAM & 11 & 51 & 25.5 & 371 & 23.5 & & & & & 1 & & & & 31 & 30 & & 24 & $14 c$ \\
\hline 35. GOOD SPIRIT L. & 3 & 3 & 1.5 & 102 & 4.2 & 4 & 2 & 3 & 5 & 20 & 40 & 2 & 5 & 20 & 2 & 1 & & \\
\hline 36. GOVENLOCK & 10 & 16 & 8 & 300 & 13 & & & & & & & 50 & & 25 & 25 & & & \\
\hline 37. GRASSLANDS & 7 & 1.5 & 0.75 & 200 & 7.5 & & & & & & & 50 & & 25 & 20 & 5 & & \\
\hline 38. GRAYSON & 3 & 1 & 1 & 50 & 6 & 0.5 & & & & 40 & & & & 5 & $20 \mid$ & 5 & 30 & \\
\hline 39. HARRIS & 1 & 6 & 2.75 & 178 & 6 & & & & & 23 & & 6 & & 30 & 30 & 11 & & \\
\hline 40. HEPBUAN & 3 & 1 & 1 & 45 & 2 & 2 & & & & 10 & & & & 30 & 20 & 40 & & \\
\hline 41. HOLBIEN & 2 & 1 & 1 & & & 3 & $\overline{5}$ & 25 & 20 & 0 & 15 & 0 & 0 & 10 & 25 & & & \\
\hline 42. HORSESHOE B. & 2 & 1.5 & 1 & & & 2 & & 20 & & & & & 10 & & 70 & & & \\
\hline 43. HUMBOLDT & 2 & 6 & 3 & 50 & 5 & & & & & 70 & & & & & 20 & 10 & & \\
\hline $\begin{array}{l}\text { 44. INDIAN HEAD } \\
\text { 45. KAMSACK }\end{array}$ & $\begin{array}{l}20 \\
22\end{array}$ & 12 & 9 & 185 & 12 & 10 & & & & 20 & 20 & 5 & & 20 & 20 & 10 & 5 & \\
\hline 46. KELVNGTON & 3 & & & 60 & 2.5 & 6 & & & & 15 & & & & 25 & 60 & & & \\
\hline 47. KENASTON & 2 & 2 & 1 & $1 œ 2$ & 6 & 2 & & & & 10 & 5 & & 25 & 40 & 15 & 5 & & \\
\hline 48. KENOSEE LAKE & 2 & 1 & 1 & 58 & 3 & & & & 20 & & & & & & & 80 & & \\
\hline 49. KILWNNING & 3 & 1 & 0.5 & 120 & 5 & & 2 & 5 & 5 & 30 & 10 & & 8 & 9 & 20 & 1 & & \\
\hline 50. KINLOCH & 9 & 9 & 3.5 & 96 & 2 & 5 & 35 & 35 & 15 & 10 & & & & & 5 & & & \\
\hline 51. KUTAWAGANL & 3 & 2 & 1 & 200 & 5 & & & & & 20 & 10 & 10 & & 40 & 10 & 10 & & \\
\hline 52. KME & 2 & & & 115 & 4 & & & & & & 10 & 15 & & 70 & 5 & & & \\
\hline 53. LARONGE & 2 & 5 & 1 & 70 & 4 & 0.5 & & 45 & & & & & & & & 50 & 5 & \\
\hline 54. LAST MT. L. & 3 & 3 & 2 & 215 & 5 & & & & & 10 & & 30 & & 50 & 5 & 4 & 1 & \\
\hline 55. LEADER (North) & 1 & 4 & 3 & 20 & 2 & 1 & & & & & & 50 & & 30 & 10 & 10 & & \\
\hline
\end{tabular}




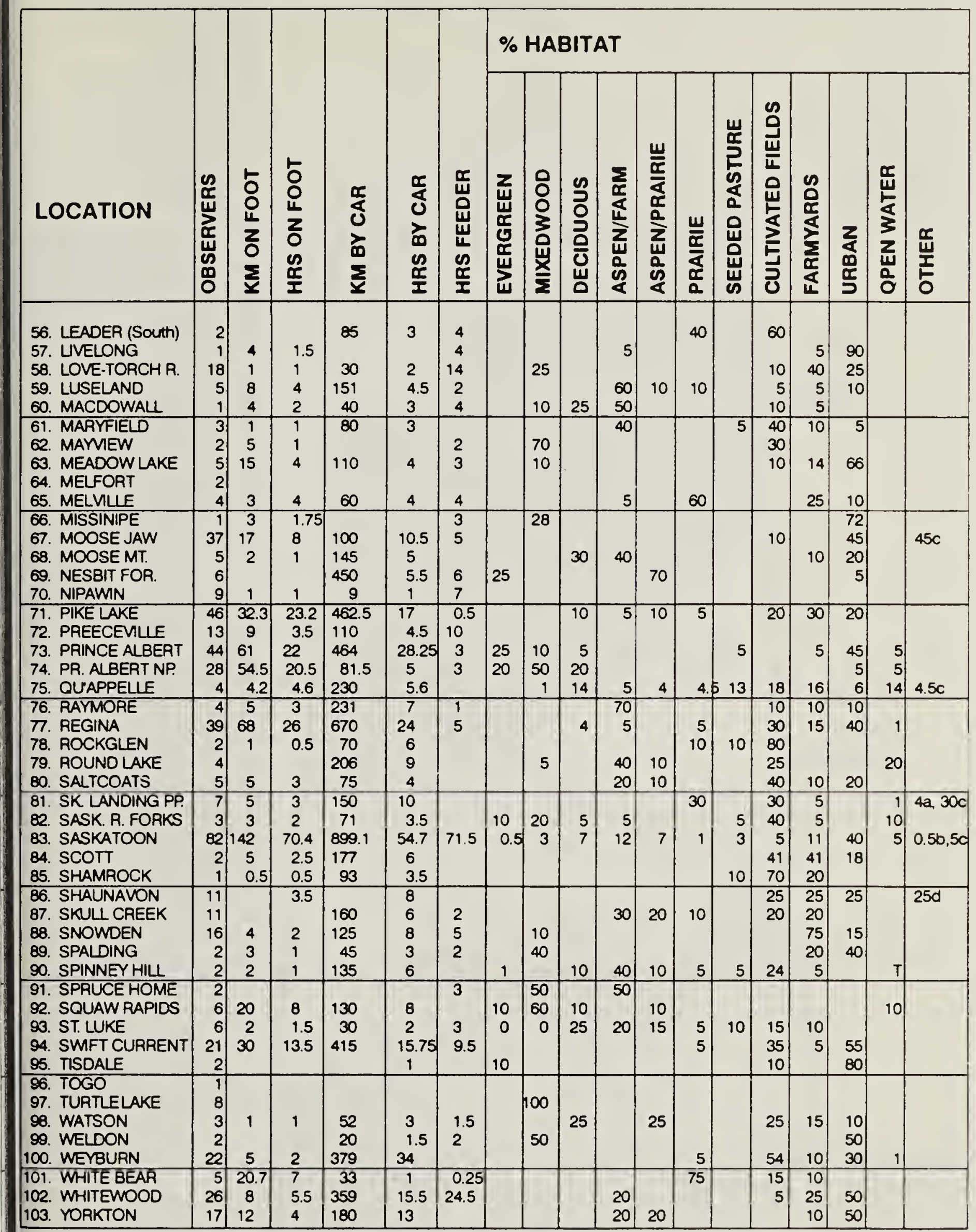

- frozen lake; ${ }^{b}$ garbage dump; ${ }^{c}$ riparian 

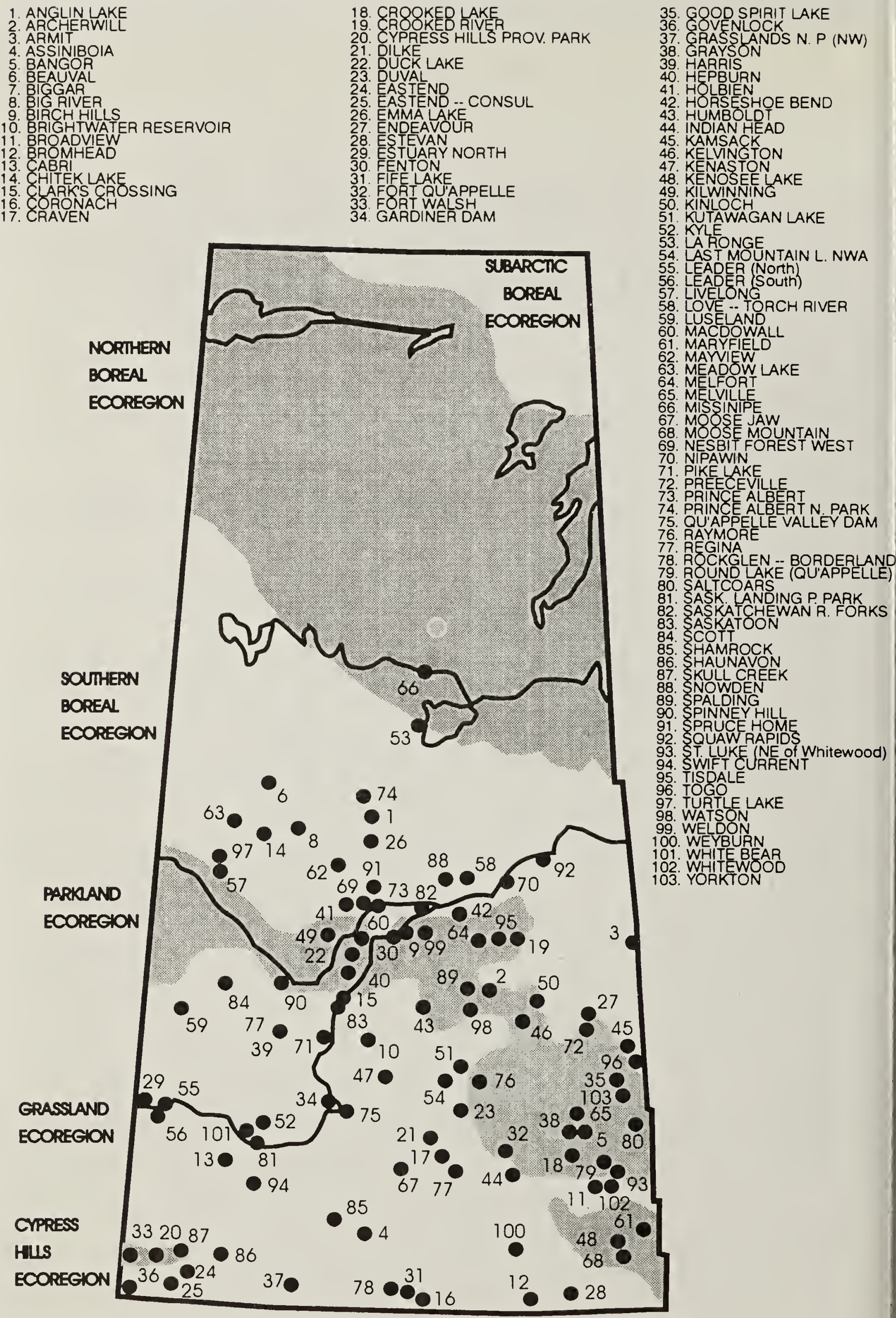

Figure 1. Location of 1997 counts (numbers correspond to locality names in Tables). 
Table 3-1. SPECIES RECORDED FROM MORE THAN NINE LOCALITIES $(+=$ species seen during the count period but not on count day)

SPECIES

CANADA GOOSE

MALLARD

COMMON GOLDENEYE

BALD EAGLE

NORTHERN GOSHAWK

ROUGH-LEGGED HAWK

GOLDEN EAGLE

MERLIN

GRAY PARTRIDGE

RING-NECKED PHEASANT

RUFFED GROUSE

SHARP-TAILED GROUSE

ROCK DOVE

GREAT HORNED OWL

SNOWY OWL

DOWNY WOODPECKER

HAIRY WOODPECKER

BLACK-BACKED WOODPECKER

NORTHERN FLICKER (Y-S)

PILEATED WOODPECKER

HORNED LARK

GRAY JAY

BLUE JAY

BLACK-BILLED MAGPIE

COMMON RAVEN

BLACK-CAPPED CHICKADEE

BOREAL CHICKADEE

RED-BREASTED NUTHATCH

WHITE-BREASTED NUTHATCH

GOLDEN-CROWNED KINGLET

AMERICAN ROBIN

BOHEMIAN WAXWING

CEDAR WAXWING

NORTHERN SHRIKE

EUROPEAN STARLING

DARK-EYED JUNCO (S-C

SNOW BUNTING

PINE GROSBEAK

PURPLE FINCH

HOUSE FINCH

COMMON REDPOLL

HOARY REDPOLL

PINE SISKIN

EVENING GROSBEAK

HOUSE SPARROW

TOTAL INDIVIDUALS COUNT DAY

NO. SPECIES COUNT DAY

NO. SPECIES COUNT PERIOD

NO. INDIVIDUALS IN TABLES 4 \& 5

NO. SPECIES IN TABLES 4 \& 5
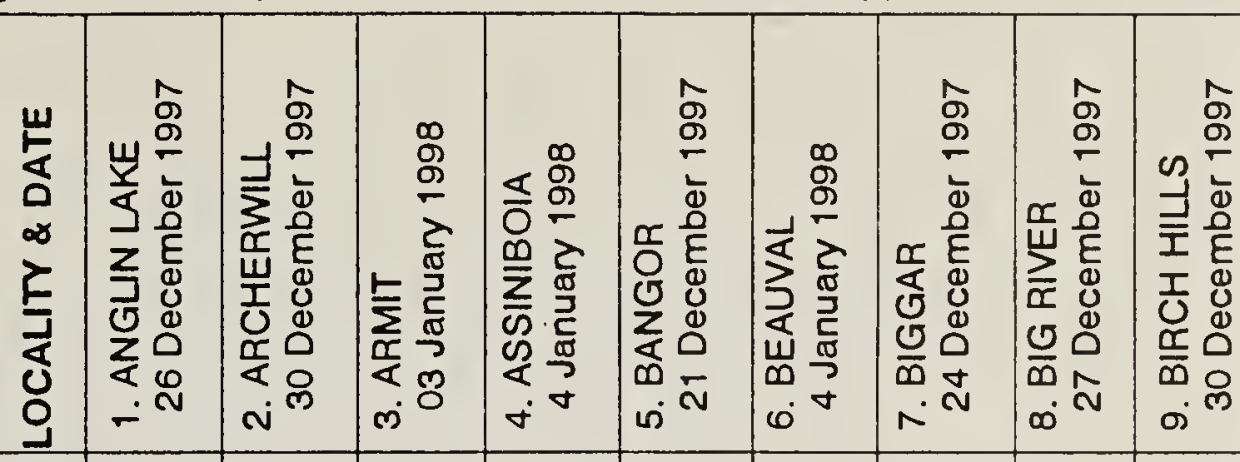

3

\begin{tabular}{|l|r|r|r|r|r|r|r|r|}
\hline & & & & & & 4 & & \\
\hline & & & & & & & & \\
\hline & & & & + & & & & \\
\hline & & 2 & & & 1 & & & \\
\hline & & 1 & & + & & & & \\
\hline & & & 1 & & & 2 & & \\
\hline & & & 29 & & & & & 22 \\
\hline
\end{tabular}

\begin{tabular}{|r|r|r|r|r|r|r|r|r|}
\hline 1 & 7 & 20 & & 3 & 1 & & + & 1 \\
\hline & & 24 & 18 & 4 & + & 93 & & \\
\hline & & & & 8 & & 155 & & 21 \\
\hline & & 1 & 2 & & 1 & 2 & & 1 \\
\hline & & & 1 & & & & & 2 \\
\hline 3 & 6 & 2 & & 8 & 1 & 2 & & 4 \\
\hline 4 & 8 & 4 & & 4 & 3 & & 1 & 3 \\
\hline
\end{tabular}

\begin{tabular}{|l|r|r|l|l|l|l|l|l}
\hline & & 1 & & 4 & 3 & & 1 & 3 \\
\hline & & & & & & & & \\
\hline & & 1 & & & 1 & & & \\
\hline
\end{tabular}

\begin{tabular}{rrrr|r|r|r|r|r}
\hline 8 & 4 & 11 & & & 5 & & 2 & \\
\hline 9 & 30 & 4 & 2 & & 12 & 2 & 2 & \\
\hline & 11 & 28 & 48 & 38 & 27 & 155 & 1 & 65 \\
\hline 11 & 7 & 74 & & 37 & 48 & 2 & 1 & 7 \\
\hline 22 & 31 & 31 & 2 & 60 & 13 & 25 & 3 & 34 \\
\hline 5 & 4 & 12 & & & 1 & 3 & 2 & \\
\hline
\end{tabular}

\begin{tabular}{r|r|r|l|r|r|r|r|r}
\hline 5 & 4 & 12 & & & 1 & 3 & 2 & \\
\hline 2 & 3 & 1 & & 4 & 1 & 8 & 3 & \\
\hline & 2 & 1 & & & & & 2 & \\
\hline & & & & & & 5 & &
\end{tabular}

\begin{tabular}{|r|r|r|r|r|r|r|r|r|}
\hline & & & & & & & & \\
\hline & & & & + & & 2 & & 177 \\
\hline & & & & & & & & \\
\hline & & & & & & & & \\
\hline & & & & & & 27 & & 6 \\
\hline
\end{tabular}

\begin{tabular}{|r|r|r|r|r|r|r|r|r|}
\hline & & 155 & 6 & + & 2 & & & 13 \\
\hline 5 & 25 & 41 & & 21 & 18 & 2 & 2 & 8 \\
\hline & & & & & + & & & \\
\hline & & & & & & & & \\
\hline & 4 & 232 & & 45 & 11 & 49 & & 7 \\
\hline & & 3 & & & & & & \\
\hline 20 & 297 & 16 & & & & & & \\
\hline & 32 & 7 & 270 & 42 & & 610 & & 230 \\
\hline 91 & 471 & 675 & 419 & 294 & 197 & 1151 & 19 & 605 \\
\hline 12 & 15 & 25 & 11 & 13 & 20 & 21 & 10 & 18 \\
\hline 12 & 15 & 25 & 11 & 17 & 24 & 21 & 11 & 18 \\
\hline 1 & 0 & 3 & 40 & 0 & 4 & 2 & 0 & 1 \\
\hline 1 & 0 & 3 & 1 & 0 & 5 & 2 & 0 & 1 \\
\hline
\end{tabular}


Table 3-2. SPECIES RECORDED FROM MORE THAN NINE LOCALITIES $(t=$ species seen during the count period but not on count day)

\begin{tabular}{|c|c|c|c|c|c|c|c|c|c|}
\hline 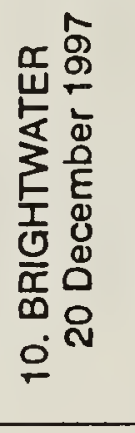 & 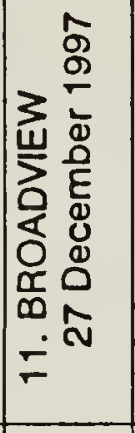 & 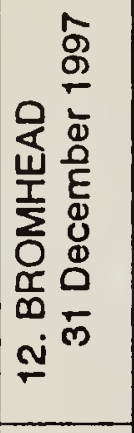 & 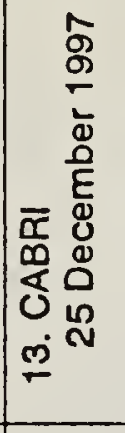 & 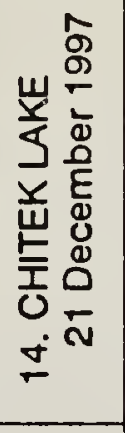 & 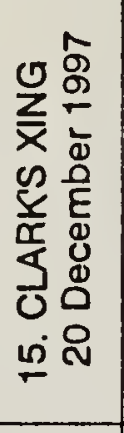 & 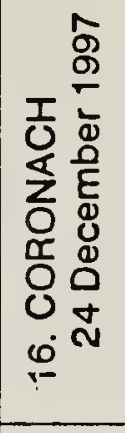 & 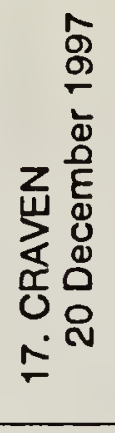 & 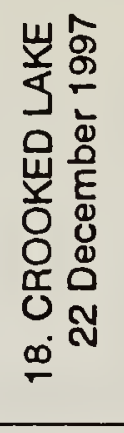 & 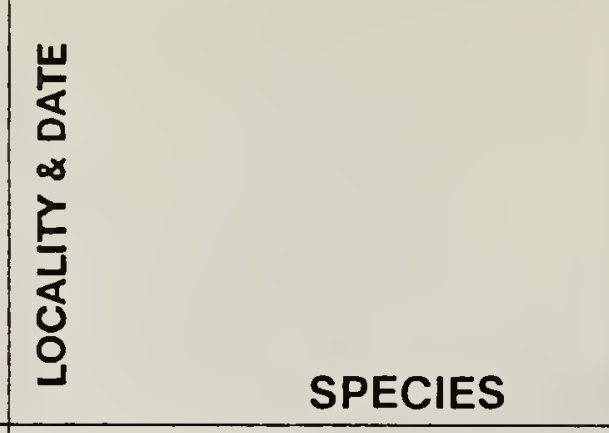 \\
\hline & & & & & 36 & 5700 & & 258 & CANADA GOOSE \\
\hline & & & & & 1 & 17000 & 7 & 297 & MALLARD \\
\hline & & & & & 51 & 8 & & & COMMON GOLDENEYE \\
\hline 2 & 1 & & & & & & & 3 & BALD EAGLE \\
\hline & & & & & & & & & NORTHERN GOSHAWK \\
\hline & & & 1 & & & & & & ROUGH-LEGGED HAWK \\
\hline 1 & & & & & & 5 & 2 & 4 & GOLDEN EAGLE \\
\hline & & & & & 1 & & & & MERLIN \\
\hline & & & 17 & & 28 & & 10 & & GRAY PARTRIDGE \\
\hline & & 1 & & & & & & & RING-NECKED PHEASANT \\
\hline & 2 & & & & & & 1 & 1 & RUFFED GROUSE \\
\hline 13 & & 37 & & & 15 & & 8 & & SHARP-TAILED GROUSE \\
\hline 43 & 61 & & 26 & & 182 & 45 & 70 & 110 & ROCK DOVE \\
\hline$t$ & 1 & 2 & 3 & & 2 & & 1 & & GREAT HORNED OWL \\
\hline 1 & + & & 5 & & 7 & 1 & 3 & 1 & SNOWY OWL \\
\hline 1 & 3 & & & & 5 & & 4 & 1 & DOWNY WOODPECKER \\
\hline 2 & 4 & & & 1 & 3 & & 7 & 2 & HAIRY WOODPECKER \\
\hline & & & & & & & & & BLACK-BACKED WOODPECKER \\
\hline & & & & & 1 & & & & NORTHERN FLICKER $(\gamma-S)$ \\
\hline & & & & & & & & & PILEATED WOODPECKER \\
\hline & & 2 & & & & 1 & & & HORNED LARK \\
\hline & & & & 2 & & & & & GRAY JAY \\
\hline & 2 & & & 4 & 1 & & 33 & 7 & BLUE JAY \\
\hline 61 & 47 & 2 & 9 & 33 & 358 & 10 & 141 & 34 & BLACK-BILLED MAGPIE \\
\hline 8 & 22 & & & 116 & 15 & & & 10 & COMMON RAVEN \\
\hline 14 & 35 & & & 14 & 97 & & 84 & 104 & BLACK-CAPPED CHICKADEE \\
\hline & & & & 7 & & & & & BOREAL CHICKADEE \\
\hline 1 & 7 & & 1 & & & & 5 & 2 & RED-BREASTED NUTHATCH \\
\hline & 2 & & & & & & 9 & 10 & WHITE-BREASTED NUTHATCH \\
\hline 2 & & & & & & & & & GOLDEN-CROWNED KINGLET \\
\hline & & & & & 5 & & 3 & + & AMERICAN ROBIN \\
\hline & 173 & & & & 553 & & 22 & 140 & BOHEMIAN WAXWING \\
\hline & & & & & 21 & & & & CEDAR WAXWING \\
\hline & & & & & & & & 2 & NORTHERN SHRIKE \\
\hline & & & & & 34 & & & & EUROPEAN STARLING \\
\hline & & & & & 1 & & & & DARK-EYED JUNCO (S-C) \\
\hline 1 & 3 & & & 1 & 32 & 62 & & & SNOW BUNTING \\
\hline 9 & 14 & & & 6 & 54 & & 4 & 3 & PINE GROSBEAK \\
\hline & & & & & & & & + & PURPLE FINCH \\
\hline & 1 & & & & & & & & HOUSE FINCH \\
\hline 11 & 2 & & & & 76 & & 15 & & COMMON REDPOLL \\
\hline & & & & & & & 1 & & HOARY REDPOLL \\
\hline & 2 & & & & & & & & PINE SISKIN \\
\hline & & & & 38 & & & & & EVENING GROSBEAK \\
\hline 395 & 233 & 99 & 95 & & 2191 & 110 & 463 & 151 & HOUSE SPARROW \\
\hline 568 & 623 & 143 & 157 & 222 & 3821 & 23038 & 895 & 1145 & TOTAL INDIVIDUALS COUNT DAY \\
\hline 19 & 20 & 6 & 8 & 10 & 25 & 29 & 23 & 22 & NO. SPECIES COUNT DAY \\
\hline 20 & 21 & 6 & 8 & 10 & 25 & 29 & 23 & 25 & NO. SPECIES COUNT PERIOD \\
\hline 2 & 8 & 0 & 0 & 0 & 51 & 95 & 2 & & NO. INDIVIDUALS IN TABLES 4 \& 5 \\
\hline 2 & 1 & of & 0 & 0 & 2 & 18 & 2 & 2 & NO. SPECIES IN TABLES 4 \& 5 \\
\hline
\end{tabular}


Table 3-3. SPECIES RECORDED FROM MORE THAN NINE LOCALITIES $(t=$ species seen during the count period but not on count day)

SPECIES
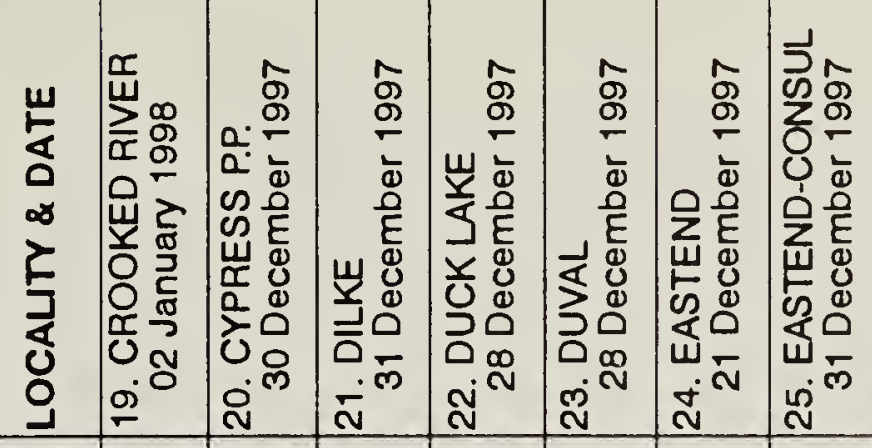

崖

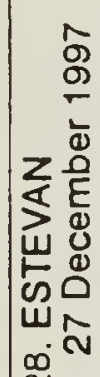

CANADA GOOSE

MALLARD

COMMON GOLDENEYE

BALD EAGLE

NORTHERN GOSHAWK

ROUGH-LEGGED HAWK

GOLDEN EAGLE

MERLIN

GRAY PARTRIDGE

RING-NECKED PHEASANT

RUFFED GROUSE

SHARP-TAILED GROUSE

ROCK DOVE

GREAT HORNED OWL

SNOWY OWL

DOWNY WOODPECKER

HAIRY WOODPECKER

BLACK-BACKED WOODPECKER

NORTHERN FLICKER (Y-s)

PILEATED WOODPECKER

HORNED LARK

GRAY JAY

BLUE JAY

BLACK-BILLED MAGPIE

COMMON RAVEN

BLACK-CAPPED CHICKADEE

BOREAL CHICKADEE

RED-BREASTED NUTHATCH

WHITE-BREASTED NUTHATCH

GOLDEN-CROWNED KINGLET

AMERICAN ROBIN

BOHEMIAN WAXWING

CEDAR WAXWING

NORTHERN SHRIKE

EUROPEAN STARLING

DARK-EYED JUNCO (S-C)

SNOW BUNTING

PINE GROSBEAK

PURPLE FINCH

HOUSE FINCH

COMMON REDPOLL

HOARY REDPOLL

PINE SISKIN

EVENING GROSBEAK

HOUSE SPARROW

TOTAL INDIVIDUALS COUNT DAY

NO. SPECIES COUNT DAY

NO. SPECIES COUNT PERIOD

NO. INDIVIDUALS IN TABLES 4 \& 5

NO. SPECIES IN TABLES 4 \& 5

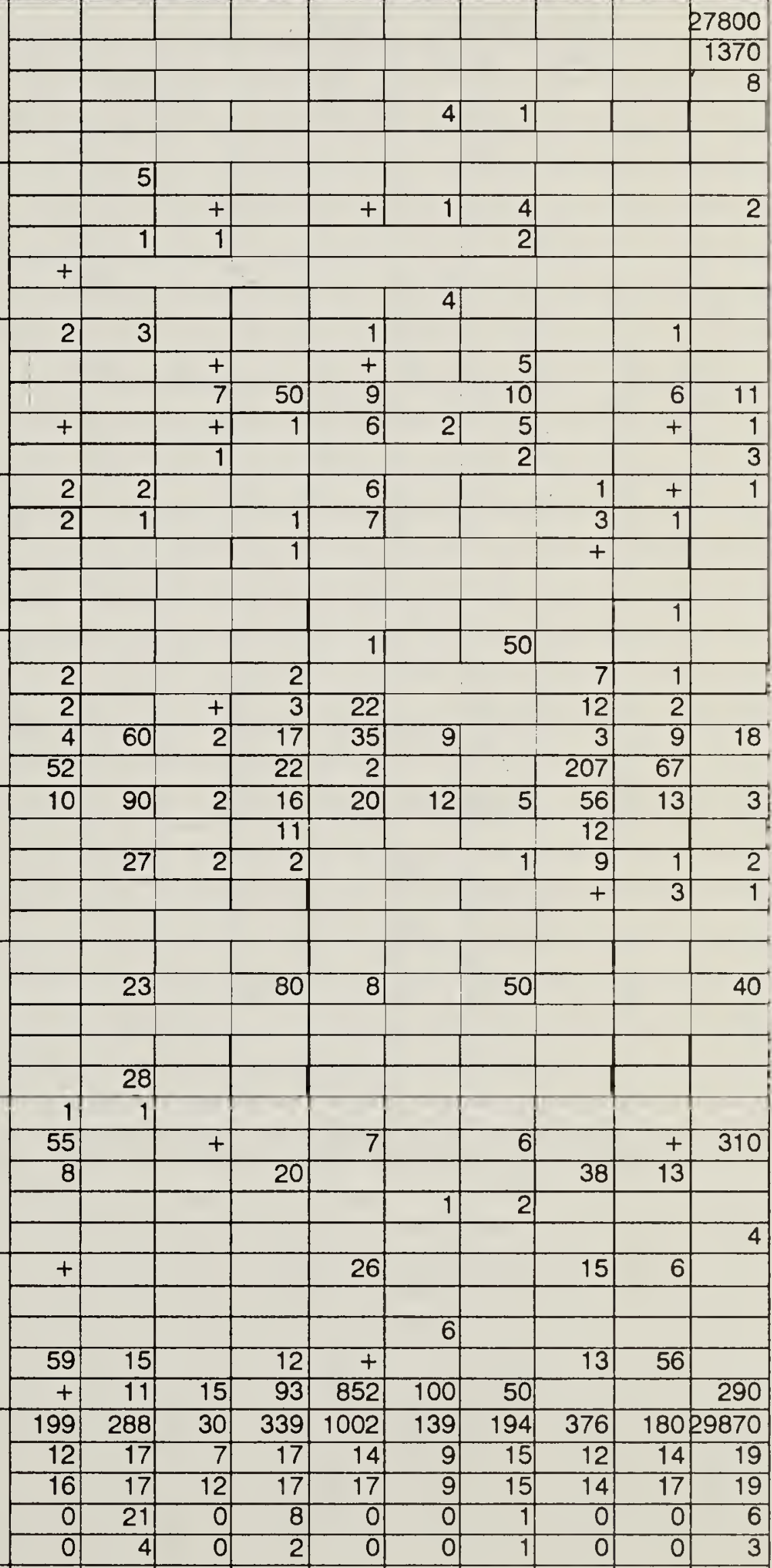


Table 3-4. SPECIES RECORDED FROM MORE THAN NINE LOCALITIES $(+=$ species seen during the count period but not on count day)

\begin{tabular}{|c|c|c|c|c|c|c|c|c|c|c|}
\hline 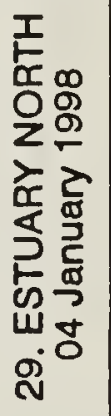 & 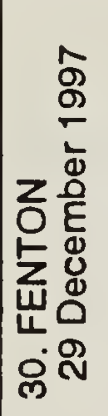 & 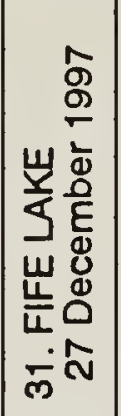 & 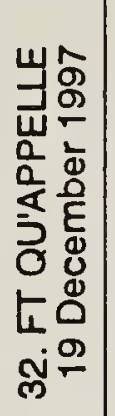 & 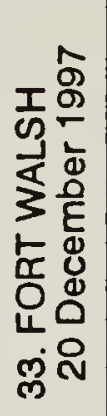 & 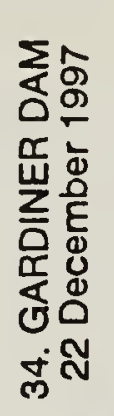 & 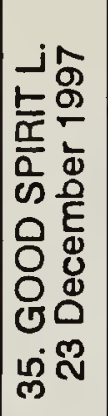 & 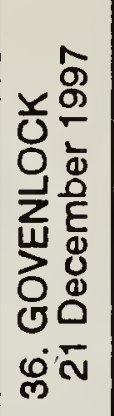 & 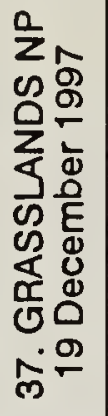 & 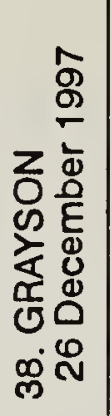 & $\begin{array}{l}\frac{1}{\alpha} \\
0 \\
\frac{1}{d} \\
\frac{J}{d} \\
0 \\
0\end{array}$ \\
\hline & & 7240 & 5375 & & 3126 & & & & & \multirow{5}{*}{$\begin{array}{l}\text { CANADA GOOSE } \\
\text { MALLARD } \\
\text { COMMON GOLDENEYE } \\
\text { BALD EAGLE } \\
\text { NORTHERN GOSHAWK }\end{array}$} \\
\hline & & 2893 & 95 & 8 & 2580 & & 4 & & 187 & \\
\hline & & 14 & 13 & & 78 & & & & & \\
\hline & 1 & 1 & 11 & 2 & 17 & & 3 & & 4 & \\
\hline & & & + & & 1 & & & & & \\
\hline & & & & 2 & & & 5 & & & \multirow{5}{*}{$\begin{array}{l}\text { ROUGH-LEGGED HAWK } \\
\text { GOLDEN EAGLE } \\
\text { MERLIN } \\
\text { GRAY PARTRIDGE } \\
\text { RING-NECKED PHEASANT }\end{array}$} \\
\hline+ & & 3 & + & 2 & 2 & & 3 & & & \\
\hline 1 & & & & & & & 1 & & & \\
\hline+ & & & + & 9 & & & + & 30 & & \\
\hline 21 & & & & 3 & & & 7 & 1 & & \\
\hline & 1 & & 1 & 5 & & 2 & & & 3 & \multirow{5}{*}{$\begin{array}{l}\text { RUFFED GROUSE } \\
\text { SHARP-TAILED GROUSE } \\
\text { ROCK DOVE } \\
\text { GREAT HORNED OWL } \\
\text { SNOWY OWL }\end{array}$} \\
\hline 11 & 30 & & & 20 & 4 & & 40 & 38 & & \\
\hline 22 & 1 & & 19 & 1 & 93 & 7 & & & & \\
\hline+ & 1 & & 1 & 1 & 10 & 1 & 7 & 3 & & \\
\hline 1 & 1 & 3 & + & 1 & 3 & & 4 & 7 & & \\
\hline 1 & 3 & & 13 & 3 & 1 & 1 & & & 3 & \multirow{5}{*}{$\begin{array}{l}\text { DOWNY WOODPECKER } \\
\text { HAIRY WOODPECKER } \\
\text { BLACK-BACKED WOODPECKER } \\
\text { NORTHERN FLICKER (Y-S) } \\
\text { PILEATED WOODPECKER }\end{array}$} \\
\hline & 4 & & 6 & 5 & 1 & 4 & & & 3 & \\
\hline & & & & & & & & & & \\
\hline & & & & & & & & 2 & & \\
\hline & & & & & & & & & & \\
\hline & + & 5 & & 1 & 1 & & 235 & & 14 & \multirow{5}{*}{$\begin{array}{l}\text { HORNED LARK } \\
\text { GRAY JAY } \\
\text { BLUE JAY } \\
\text { BLACK-BILLED MAGPIE } \\
\text { COMMON RAVEN }\end{array}$} \\
\hline & & & 1 & & & + & & & & \\
\hline 7 & + & & 22 & 1 & 12 & 2 & & 3 & 2 & \\
\hline 17 & 20 & & 51 & 159 & 221 & 10 & 18 & 44 & 26 & \\
\hline & 6 & & 11 & & 5 & 18 & & & 4 & \\
\hline 4 & 15 & & 122 & 251 & 21 & 24 & & & 27 & \multirow{5}{*}{$\begin{array}{l}\text { BLACK-CAPPED CHICKADEE } \\
\text { BOREAL CHICKADEE } \\
\text { RED-BREASTED NUTHATCH } \\
\text { WHITE-BREASTED NUTHATCH } \\
\text { GOLDEN-CROWNED KINGLET }\end{array}$} \\
\hline & & & & & & & & & & \\
\hline & & & 4 & 123 & 2 & & & & & \\
\hline & & & 14 & & & 4 & & & & \\
\hline & & & & 32 & & & & & & \\
\hline & & & 8 & 1 & 1 & & & & & \multirow{5}{*}{$\begin{array}{l}\text { AMERICAN ROBIN } \\
\text { BOHEMIAN WAXWING } \\
\text { CEDAR WAXWING } \\
\text { NORTHERN SHRIKE } \\
\text { EUROPEAN STARLING }\end{array}$} \\
\hline & 49 & & 72 & 25 & 1 & 62 & & & & \\
\hline & & & & & & & & & & \\
\hline & & & & 3 & 1 & & & & 1 & \\
\hline & & & & 1 & 52 & & 6 & & & \\
\hline & & & & 78 & 1 & & 1 & & & \multirow{5}{*}{$\begin{array}{l}\text { DARK-EYED JUNCO (S-C) } \\
\text { SNOW BUNTING } \\
\text { PINE GROSBEAK } \\
\text { PURPLE FINCH } \\
\text { HOUSE FINCH }\end{array}$} \\
\hline+ & 51 & & & 90 & 202 & & 343 & 2 & & \\
\hline & 2 & & 8 & 8 & 1 & 5 & & & & \\
\hline & & & + & & & & & & & \\
\hline & & & + & & & & & & & \\
\hline & 19 & 2 & 35 & 227 & 58 & 5 & 23 & & & \multirow{5}{*}{$\begin{array}{l}\text { COMMON REDPOLL } \\
\text { HOARY REDPOLL } \\
\text { PINE SISKIN } \\
\text { EVENING GROSBEAK } \\
\text { HOUSE SPARROW }\end{array}$} \\
\hline & & & & & 2 & & & & & \\
\hline & & & + & 80 & & & & & & \\
\hline 9 & & & + & & & & & & & \\
\hline 5 & 31 & 15 & 250 & 132 & 1036 & 120 & 458 & 15 & 50 & \\
\hline 108 & 235 & 10186 & 6148 & 1488 & 7669 & 266 & 1210 & 146 & 329 & \multirow{5}{*}{$\begin{array}{l}\text { TOTAL INDIVIDUALS COUNT DAY } \\
\text { NO. SPECIES COUNT DAY } \\
\text { NO. SPECIES COUNT PERIOD } \\
\text { NO. INDIVIDUALS IN TABLES } 4 \& 5 \\
\text { NO. SPECIES IN TABLES } 4 \& 5\end{array}$} \\
\hline 12 & 16 & 12 & 23 & 43 & 40 & 15 & 27 & 11 & 15 & \\
\hline 16 & 18 & 12 & 40 & 43 & 40 & 16 & 29 & 11 & 15 & \\
\hline 9 & 0 & 3 & 16 & 214 & 136 & 1 & 52 & 1 & 5 & \\
\hline 1 & 0 & 3 & 11 & 15 & 13 & 1 & 13 & 1 & 3 & \\
\hline
\end{tabular}


Table 3-5. SPECIES RECORDED FROM MORE THAN NINE LOCALITIES $(t=$ species seen during the count period but not on count day)

\section{SPECIES}

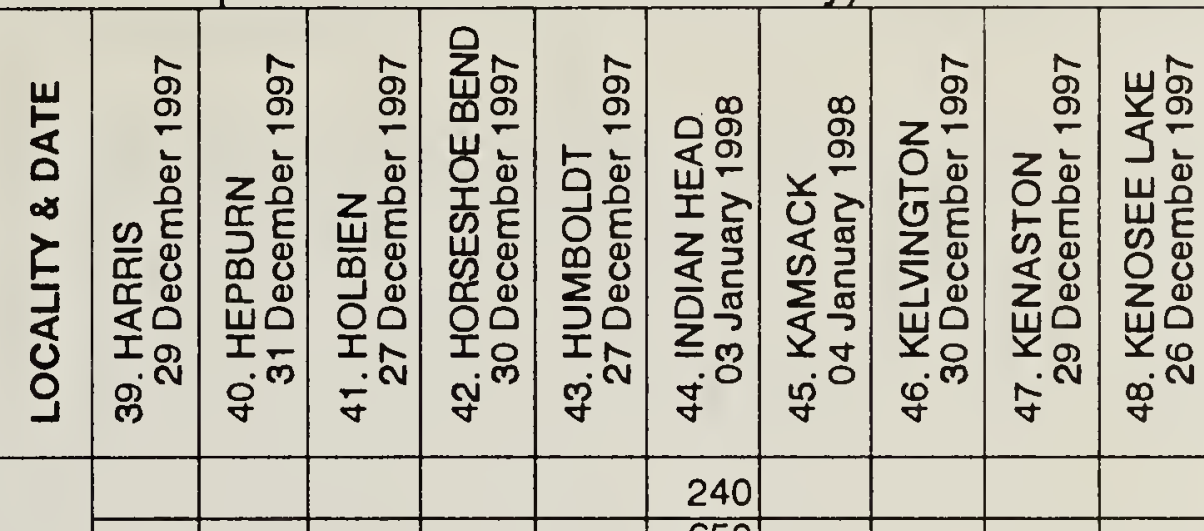

CANADA GOOSE

MALLARD

COMMON GOLDENEYE

BALD EAGLE

NORTHERN GOSHAWK

ROUGH-LEGGED HAWK

GOLDEN EAGLE

MERLIN

GRAY PARTRIDGE

RING-NECKED PHEASANT

RUFFED GROUSE

SHARP-TAILED GROUSE

ROCK DOVE

GREAT HORNED OWL

SNOWY OWL

DOWNY WOODPECKER

HAIRY WOODPECKER

BLACK-BACKED WOODPECKER

NORTHERN FLICKER (Y-S)

PILEATED WOODPECKER

HORNED LARK

GRAY JAY

BLUE JAY

BLACK-BILLED MAGPIE

COMMON RAVEN

BLACK-CAPPED CHICKADEE

BOREAL CHICKADEE

RED-BREASTED NUTHATCH

WHITE-BREASTED NUTHATCH

GOLDEN-CROWNED KINGLET

AMERICAN ROBIN

BOHEMIAN WAXWING

CEDAR WAXWING

NORTHERN SHRIKE

EUROPEAN STARLING

DARK-EYED JUNCO (S-C)

SNOW BUNTING

PINE GROSBEAK

PURPLE FINCH

HOUSE FINCH

COMMON REDPOLL

HOARY REDPOLL

PINE SISKIN

EVENING GROSBEAK

HOUSE SPARROW

TOTAL INDIVIDUALS COUNT DAY

NO. SPECIES COUNT DAY

NO. SPECIES COUNT PERIOD

NO. INDIVIDUALS IN TABLES 4 \& 5

NO. SPECIES IN TABLES 4 \& 5

\begin{tabular}{|c|c|c|c|c|c|c|c|c|c|}
\hline & & & & & 240 & & & & \\
\hline & & & & & 650 & & & & \\
\hline & & & & & & & & & \\
\hline & & & 2 & & 3 & & & & \\
\hline & & & & & & & & & \\
\hline & & & & & & & & & \\
\hline & & & & & 1 & 1 & & 1 & \\
\hline 1 & & & & & & & & & \\
\hline 16 & & 2 & & & 18 & & & + & \\
\hline & & & & & 1 & & & & \\
\hline & & & 1 & 2 & 2 & 20 & 6 & & 1 \\
\hline 88 & & & & 41 & 8 & 4 & 1 & + & \\
\hline 124 & & & & 300 & 84 & & 10 & 42 & \\
\hline 1 & & & & & 2 & & + & $t$ & 1 \\
\hline 1 & & & & + & 5 & 7 & . & 1 & \\
\hline & + & 2 & 2 & 1 & 4 & 7 & 3 & & 1 \\
\hline & + & 2 & 1 & + & 5 & 11 & 4 & & 2 \\
\hline & & & & & 4 & & & & \\
\hline & & & & & & & & & \\
\hline & & & & & & 2 & & & \\
\hline & & & & & & & & + & \\
\hline & & & 2 & & & 4 & & & \\
\hline & 4 & 3 & 3 & + & 13 & 60 & 7 & 2 & $\overline{15}$ \\
\hline 116 & 2 & 11 & 6 & 28 & 97 & 15 & 1 & 20 & 3 \\
\hline 1 & & 3 & 7 & 7 & 16 & 10 & 9 & & + \\
\hline 2 & 5 & 10 & 14 & 32 & 58 & 115 & 18 & 2 & 61 \\
\hline & & 1 & 3 & & & 2 & & & \\
\hline & & & $\overline{1}$ & 3 & 24 & 12 & & & 2 \\
\hline & & 1 & 1 & & 8 & 5 & 1 & & 7 \\
\hline & & & & & & & & & 7 \\
\hline & & & & + & 1 & & & & \\
\hline & & & & 14 & & 200 & & & 48 \\
\hline & 110 & & & & & & & & + \\
\hline & & & & & 1 & & & & \\
\hline & & & & 30 & & & & & \\
\hline & & & & & & 24 & 1 & & \\
\hline 23 & & & 6 & 1 & 475 & 100 & 350 & + & \\
\hline & & 25 & 13 & 1 & 20 & 99 & 14 & & $\overline{12}$ \\
\hline & & & & & & & & & 15 \\
\hline & & & & & & & & & 1 \\
\hline 9 & & 3 & 33 & 2 & 120 & 105 & 32 & 5 & 41 \\
\hline & & 2 & & & & & & & \\
\hline & & & 1 & & 4 & 108 & & & 28 \\
\hline & & 10 & 25 & & & 685 & 40 & & 50 \\
\hline 711 & 16 & & & 200 & 132 & 70 & 50 & 140 & 62 \\
\hline 1093 & 137 & 75 & 123 & 662 & 1999 & 1661 & 553 & 214 & 359 \\
\hline 12 & 5 & 13 & 19 & 14 & 30 & 23 & 17 & 9 & 20 \\
\hline 12 & 7 & 13 & 19 & 18 & 30 & 23 & 18 & 14 & 22 \\
\hline 0 & 0 & 0 & 2 & 0 & 3 & 2 & 6 & 1 & 2 \\
\hline 0 & o & 0 & 2 & 0 & 3 & 1 & 1 & 1 & 2 \\
\hline
\end{tabular}


Table 3-6. SPECIES RECORDED FROM MORE THAN NINE LOCALITIES $(t=$ species seen during the count period but not on count day)

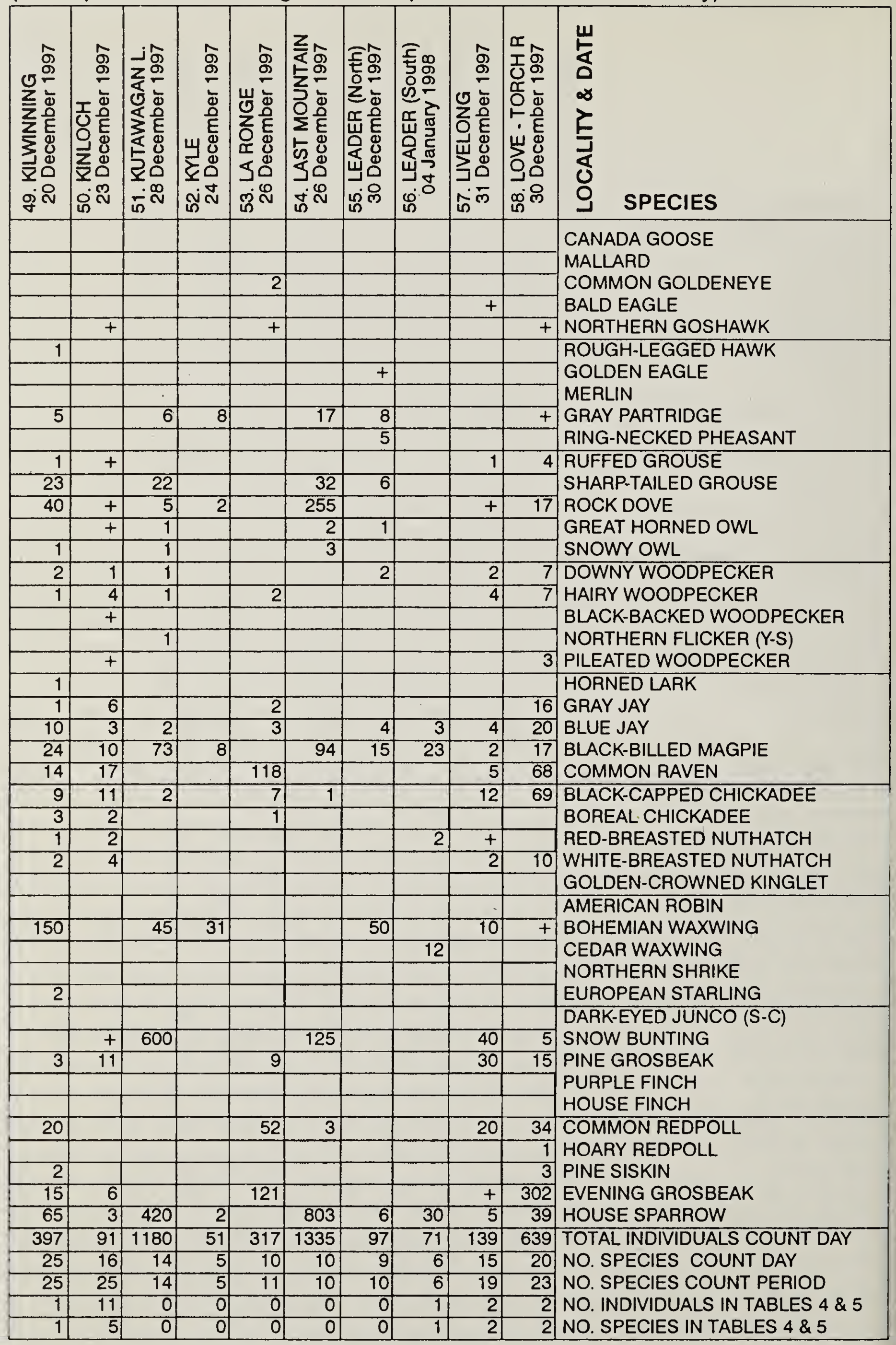


Table 3-7. SPECIES RECORDED FROM MORE THAN NINE LOCALITIES $(t=$ species seen during the count period but not on count day)

\section{SPECIES}

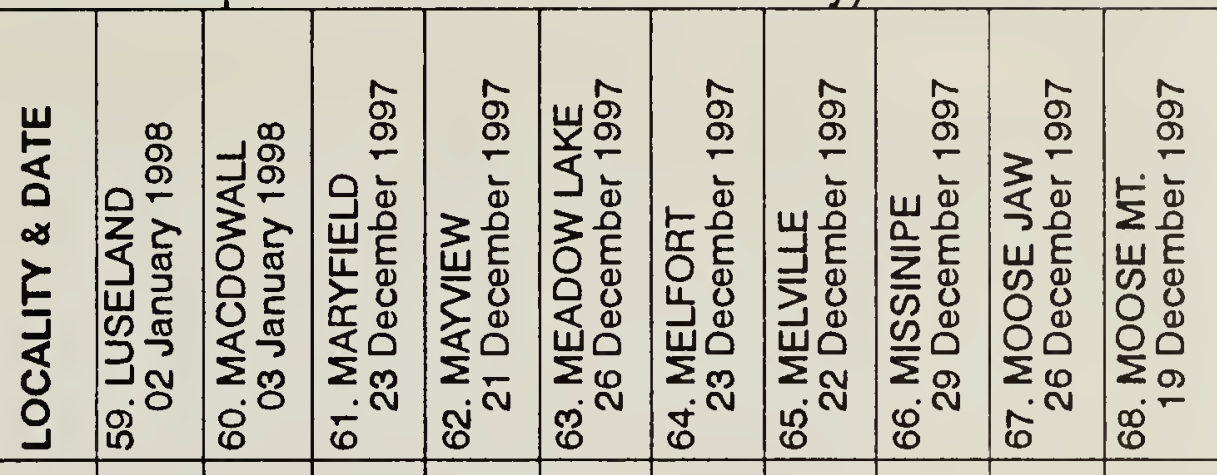

CANADA GOOSE

MALLARD

COMMON GOLDENEYE

BALD EAGLE

NORTHERN GOSHAWK

ROUGH-LEGGED HAWK

GOLDEN EAGLE

MERLIN

GRAY PARTRIDGE

RING-NECKED PHEASANT

RUFFED GROUSE

SHARP-TAILED GROUSE

ROCK DOVE

GREAT HORNED OWL

SNOWY OWL

DOWNY WOODPECKER

HAIRY WOODPECKER

BLACK-BACKED WOODPECKER

NORTHERN FLICKER $(-S)$

PILEATED WOODPECKER

HORNED LARK

GRAY JAY

BLUE JAY

BLACK-BILLED MAGPIE

COMMON RAVEN

BLACK-CAPPED CHICKADEE

BOREAL CHICKADEE

RED--BREASTED NUTHATCH

WHITE-BREASTED NUTHATCH

GOLDEN-CROWNED KINGLET

AMERICAN ROBIN

BOHEMIAN WAXWING

CEDAR WAXWING

NORTHERN SHRIKE

EUROPEAN STARLING

DARK-EYED JUNCO (S-C)

SNOW BUNTING

PINE GROSBEAK

PURPLE FINCH

HOUSE FINCH

COMMON REDPOLL

HOARY REDPOLL

PINE SISKIN

EVENING GROSBEAK

HOUSE SPARROW

TOTAL INDIVIDUALS COUNT DAY

NO. SPECIES COUNT DAY

NO. SPECIES COUNT PERIOD

NO. INDIVIDUALS IN TABLES $4 \& 5$

NO. SPECIES IN TABLES $4 \& 5$

\begin{tabular}{|c|c|c|c|c|c|c|c|c|c|}
\hline & & & & & & + & & & \\
\hline & & & & & & & & & \\
\hline & & & & & & & & & \\
\hline & & & & & & + & & & \\
\hline & & & & & & & & & \\
\hline & & & & & & & & 1 & \\
\hline & & & & & & & & & \\
\hline+ & & & & & & & & 3 & \\
\hline 21 & & 27 & & & & & & 35 & \\
\hline & & 1 & & & & & & 4 & \\
\hline & 3 & 1 & 1 & + & & & & & \\
\hline 5 & & 8 & & & & & & 5 & 4 \\
\hline 19 & 40 & 6 & & 70 & 50 & 3 & & 255 & 28 \\
\hline+ & + & 3 & & + & & 1 & & 7 & \\
\hline 2 & & & 1 & & 1 & 2 & & 3 & \\
\hline 1 & 4 & 1 & & 1 & 3 & 1 & + & 21 & 5 \\
\hline 1 & 4 & & & & & 2 & 1 & 4 & 5 \\
\hline & & & 1 & & & & & & \\
\hline+ & & & & & & & & 10 & 2 \\
\hline & + & & 1 & & & & + & & \\
\hline 1 & & 17 & & & & & & & \\
\hline 1 & 4 & & 5 & + & & & 3 & & \\
\hline$t$ & 8 & & 3 & 1 & 5 & - & 2 & 24 & 9 \\
\hline 29 & & 12 & & 33 & 10 & 5 & & 114 & 24 \\
\hline & 12 & 1 & 3 & 104 & & 2 & 1 & & \\
\hline+ & 24 & & 5 & 14 & 23 & 40 & 3 & 33 & 40 \\
\hline & & & 1 & & & 25 & 1 & & \\
\hline 1 & 2 & & 2 & 1 & 3 & & & 31 & \\
\hline & & & 1 & 1 & & & & 11 & 2 \\
\hline & & & & & & & & & \\
\hline+ & & 1 & & & & & & 1 & \\
\hline 97 & & & & 70 & 30 & & & & \\
\hline & & & & & & 70 & & & \\
\hline & & & & & & & & & \\
\hline & & & & & & & & 39 & \\
\hline & & & & & & & & & \\
\hline 425 & + & 10 & & & & & & 4 & \\
\hline & 6 & & 12 & 15 & & 16 & 3 & 6. & 14 \\
\hline & & & & & & & & 7 & 8 \\
\hline & & & & & & & & 40 & \\
\hline 28 & 6 & & & 39 & & 25 & 4 & & 70 \\
\hline & & & & & & & & & \\
\hline & & & & & & & + & 40 & 12 \\
\hline & 6 & & 23 & 67 & & 1 & 6 & & \\
\hline 94 & 1 & 40 & & 79 & 25 & 30 & & 396 & 64 \\
\hline 725 & 120 & 128 & 59 & 495 & 150 & 223 & 24 & 1096 & 288 \\
\hline 14 & 13 & 13 & 13 & 13 & 9 & 14 & 9 & 26 & 15 \\
\hline 20 & 17 & 13 & 13 & 16 & 9 & 16 & 12 & 27 & 15 \\
\hline 0 & 0 & 0 & 0 & 0 & 0 & 0 & 0 & 2 & i \\
\hline 0 & 1 & 0 & 0 & 0 & 0 & 0 & 0 & 3 & 1 \\
\hline
\end{tabular}


Table 3-8. SPECIES RECORDED FROM MORE THAN NINE LOCALITIES $(+=$ species seen during the count period but not on count day)

\begin{tabular}{|c|c|c|c|c|c|c|c|c|c|c|}
\hline 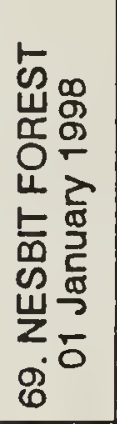 & 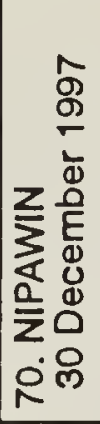 & 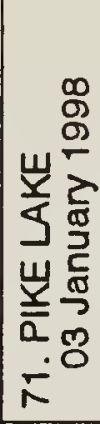 & 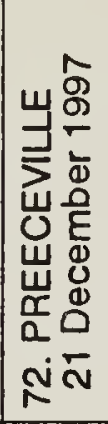 & 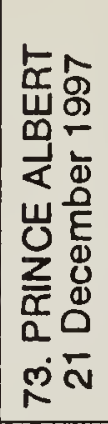 & 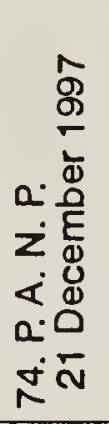 & 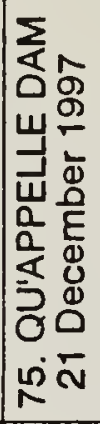 & 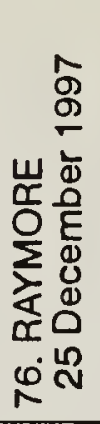 & 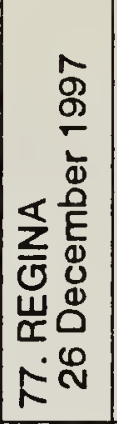 & 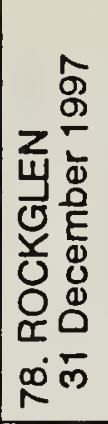 & 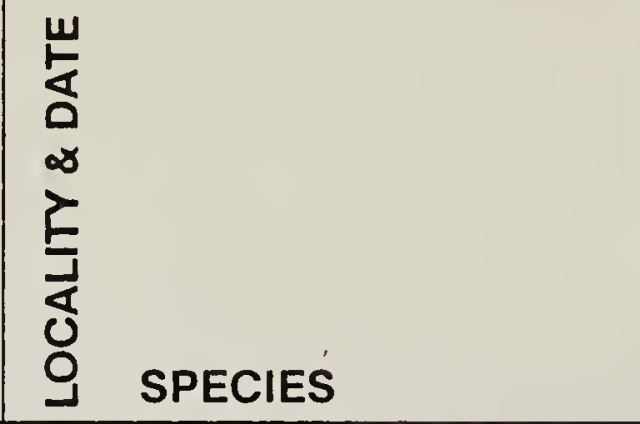 \\
\hline & & & & & & 2786 & & 4147 & & \multirow{5}{*}{$\begin{array}{l}\text { CANADA GOOSE } \\
\text { MALLARD } \\
\text { COMMON GOLDENEYE } \\
\text { BALD EAGLE } \\
\text { NORTHERN GOSHAWK }\end{array}$} \\
\hline & & & & & & 2526 & & 70 & & \\
\hline & & & & $\overline{3}$ & & 35 & & & & \\
\hline & & + & t & 1 & 2 & 11 & & & & \\
\hline \multirow[t]{5}{*}{1} & & & & & & & + & & & \\
\hline & & 1 & 1 & & & & & & 1 & ROUGH-LEGGED HAWK \\
\hline & & 1 & & & & 1 & & & + & \multirow{2}{*}{$\begin{array}{l}\text { GOLDEN EAGLE } \\
\text { MERLIN }\end{array}$} \\
\hline & & & & & & & + & 1 & & \\
\hline & & 10 & & & & & + & 32 & t & \multirow{2}{*}{$\begin{array}{l}\text { GRAY PARTRIDGE } \\
\text { RING-NECKED PHEASANT }\end{array}$} \\
\hline \multirow[t]{4}{*}{1} & & & & & & & & 2 & & \\
\hline & + & 4 & 4 & 5 & 17 & & 3 & & & RUFFED GROUSE \\
\hline & & 98 & & 1 & & & + & & 104 & \multirow{2}{*}{$\begin{array}{l}\text { SHARP-TAILED GROUSE } \\
\text { ROCK DOVE }\end{array}$} \\
\hline & & 60 & 3 & 1035 & & 16 & 7 & 717 & & \\
\hline \multirow[t]{2}{*}{1} & & 3 & & 3 & & 1 & 1 & 13 & & GREAT HORNED OWL \\
\hline & & + & & + & & 6 & + & 36 & 1 & SNOWY OWL \\
\hline 1 & 1 & 19 & 7 & 11 & & 2 & + & 9 & & \multirow{5}{*}{$\begin{array}{l}\text { DOWNY WOODPECKER } \\
\text { HAIRY WOODPECKER } \\
\text { BLACK-BACKED WOODPECKER } \\
\text { NORTHERN FLICKER (Y-S) } \\
\text { PILEATED WOODPECKER }\end{array}$} \\
\hline 1 & 1 & 20 & 9 & 15 & 1 & 2 & 1 & & & \\
\hline & & & & & 3 & & & 1 & & \\
\hline & & 2 & & 3 & & & & 14 & & \\
\hline & & & 1 & + & 2 & & & & & \\
\hline & & 2 & & + & & & & & 48 & \multirow{5}{*}{$\begin{array}{l}\text { HORNED LARK } \\
\text { GRAY JAY } \\
\text { BLUE JAY } \\
\text { BLACK-BILLED MAGPIE } \\
\text { COMMON RAVEN } \\
\end{array}$} \\
\hline & $\begin{array}{c}t \\
t\end{array}$ & & & 9 & 8 & & & & & \\
\hline 1 & 14 & 47 & 5 & 17 & & 1 & & 7 & & \\
\hline 22 & 2 & 214 & 18 & 139 & 17 & 54 & 28 & 211 & 16 & \\
\hline 41 & 38 & 26 & 79 & 307 & 17 & 13 & 2 & & & \\
\hline \multirow[t]{11}{*}{6} & 3 & 228 & 30 & 199 & 14 & 11 & 7 & 63 & & \multirow{5}{*}{$\begin{array}{l}\text { BLACK-CAPPED CHICKADEE } \\
\text { BOREAL CHICKADEE } \\
\text { RED-BREASTED NUTHATCH } \\
\text { WHITE-BREASTED NUTHATCH } \\
\text { GOLDEN-CROWNED KINGLET }\end{array}$} \\
\hline & & & & 6 & 28 & & & & & \\
\hline & 4 & 9 & 1 & 16 & 3 & & & 123 & & \\
\hline & & 4 & 2 & 5 & 1 & & & 15 & & \\
\hline & & & & 2 & & & & 18 & & \\
\hline & 1 & 14 & & 2 & & & + & + & & \multirow{5}{*}{$\begin{array}{l}\text { AMERICAN ROBIN } \\
\text { BOHEMIAN WAXWING } \\
\text { CEDAR WAXWING } \\
\text { NORTHERN SHRIKE } \\
\text { EUROPEAN STARLING } \\
\end{array}$} \\
\hline & 70 & 2904 & & 6256 & & 105 & 51 & 30 & & \\
\hline & & 20 & & & & & & 10 & & \\
\hline & & 2 & & 1 & & & & & & \\
\hline & & & & & & & 1 & 46 & & \\
\hline & & 1 & 1 & 8 & & & 1 & 14 & & \multirow{5}{*}{$\begin{array}{l}\text { DARK-EYED JUNCO (S-C) } \\
\text { SNOW BUNTING } \\
\text { PINE GROSBEAK } \\
\text { PURPLE FINCH } \\
\text { HOUSE FINCH }\end{array}$} \\
\hline 300 & & 391 & & 88 & & 1 & 13 & & 21 & \\
\hline 44 & + & 100 & 17 & 36 & 2 & $\overline{4}$ & 7 & & & \\
\hline & & & & & & & & & & \\
\hline & & & & & & 2 & & 40 & & \\
\hline 227 & & 102 & 56 & 32 & & & 3 & 25 & 27 & \multirow{5}{*}{$\begin{array}{l}\text { COMMON REDPOLL } \\
\text { HOARY REDPOLL } \\
\text { PINE SISKIN } \\
\text { EVENING GROSBEAK } \\
\text { HOUSE SPARROW }\end{array}$} \\
\hline & & & & & & & & 3 & & \\
\hline & & & & + & & & & 124 & & \\
\hline 45 & 40 & 2 & 8 & 49 & 1 & 2 & & & & \\
\hline & 85 & 398 & 12 & 327 & & 352 & 211 & 1317 & & \\
\hline 691 & 261 & 4687 & 255 & 8585 & 119 & 5934 & 339 & 7119 & 220 & \multirow{5}{*}{$\begin{array}{l}\text { TOTAL INDIVIDUALS COUNT DAY } \\
\text { NO. SPECIES COUNT DAY } \\
\text { NO. SPECIES COUNT PERIOD } \\
\text { NO. INDIVIDUALS IN TABLES } 4 \text { \& } 5 \\
\text { NO. SPECIES IN TABLES } 4 \text { \& } 5\end{array}$} \\
\hline 13 & 12 & 30 & 18 & 29 & 15 & 23 & 16 & 33 & & \\
\hline 13 & 16 & 32 & 19 & 34 & 15 & 23 & 23 & 34 & 14 & \\
\hline 0 & 2 & 5 & 1 & 9 & 3 & 3 & 3 & 31 & 1 & \\
\hline of & 2 & 3 & 1 & 3 & 1 & 3 & 2 & 9 & 4 & \\
\hline
\end{tabular}


Table 3-9. SPECIES RECORDED FROM MORE THAN NINE LOCALITIES $(t=$ species seen during the count period but not on count day)

\begin{tabular}{|c|c|c|c|c|c|c|c|c|c|c|}
\hline 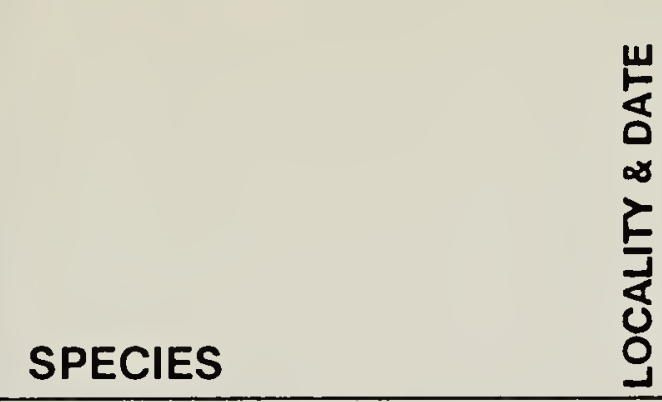 & 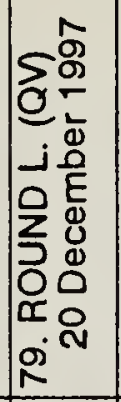 & 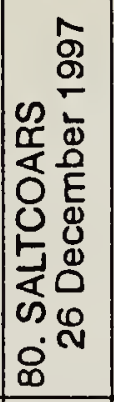 & 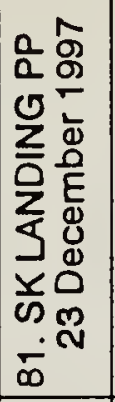 & 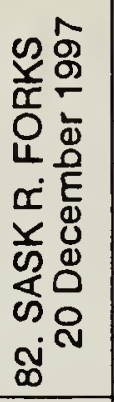 & 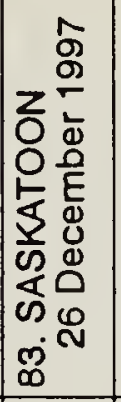 & 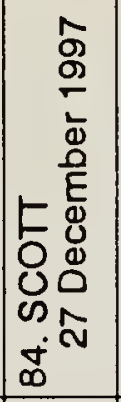 & 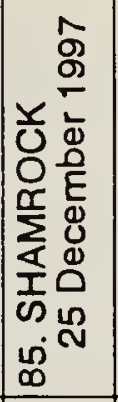 & 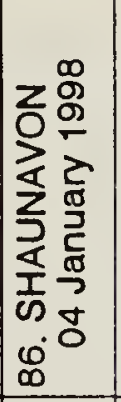 & 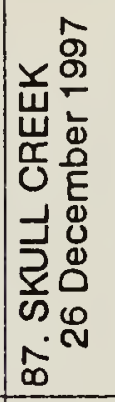 & 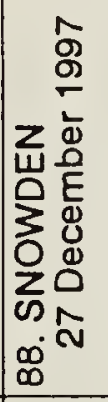 \\
\hline \multirow{5}{*}{$\begin{array}{l}\text { CANADA GOOSE } \\
\text { MALLARD } \\
\text { COMMON GOLDENEYE } \\
\text { BALD EAGLE } \\
\text { NORTHERN GOSHAWK }\end{array}$} & & & & & + & & & & 30 & \\
\hline & 6 & & 104 & & 27 & & & & 15 & \\
\hline & & & & & 485 & & & & & \\
\hline & 2 & & + & & 3 & & & & 3 & \\
\hline & 1 & & & & & & & & 1 & \\
\hline \multirow{5}{*}{$\begin{array}{l}\text { ROUGH-LEGGED HAWK } \\
\text { GOLDEN EAGLE } \\
\text { MERLIN } \\
\text { GRAY PARTRIDGE } \\
\text { RING-NECKED PHEASANT }\end{array}$} & & & & & & & & & 1 & \\
\hline & 2 & & 1 & & & & & 1 & 1 & \\
\hline & & & & & 6 & & & 1 & 1 & \\
\hline & & & 5 & & 32 & 22 & 17 & 2 & 20 & \\
\hline & & & & & + & & & & 5 & \\
\hline \multirow{5}{*}{$\begin{array}{l}\text { RUFFED GROUSE } \\
\text { SHARP-TAILED GROUSE } \\
\text { ROCK DOVE } \\
\text { GREAT HORNED OWL } \\
\text { SNOWY OWL }\end{array}$} & + & & & 1 & 1 & & & & & 6 \\
\hline & & & 4 & & 30 & & & & 59 & 5 \\
\hline & 38 & & 2 & 5 & 2433 & 12 & & 3 & & 30 \\
\hline & 2 & 1 & 1 & & 5 & 2 & & 4 & 4 & \\
\hline & & & 1 & & 1 & 2 & & 3 & 3 & \\
\hline \multirow{5}{*}{$\begin{array}{l}\text { DOWNY WOODPECKER } \\
\text { HAIRY WOODPECKER } \\
\text { BLACK-BACKED WOODPECKER } \\
\text { NORTHERN FLICKER (Y-S) } \\
\text { PILEATED WOODPECKER }\end{array}$} & 3 & 4 & & 2 & 21 & & & & 6 & 7 \\
\hline & 3 & 6 & & 1 & 25 & & & 1 & 7 & 7 \\
\hline & & & & & & & & & & \\
\hline & & & & & 14 & + & & 1 & 2 & \\
\hline & & & & 1 & & & & & & + \\
\hline \multirow{5}{*}{$\begin{array}{l}\text { HORNED LARK } \\
\text { GRAY JAY } \\
\text { BLUE JAY } \\
\text { BLACK-BILLED MAGPIE } \\
\text { COMMON RAVEN }\end{array}$} & & & 1 & & & & 4 & & 20 & \\
\hline & & & & 1 & & & & & & 5 \\
\hline & 19 & 2 & & 3 & 84 & 3 & & 8 & 6 & 13 \\
\hline & 73 & 15 & 48 & 22 & 822 & 54 & 6 & 18 & 42 & 35 \\
\hline & 10 & 9 & & 24 & 13 & 1 & & & & 201 \\
\hline \multirow{5}{*}{$\begin{array}{l}\text { BLACK-CAPPED CHICKADEE } \\
\text { BOREAL CHICKADEE } \\
\text { RED-BREASTED NUTHATCH } \\
\text { WHITE-BREASTED NUTHATCH } \\
\text { GOLDEN-CROWNED KINGLET }\end{array}$} & 65 & 25 & 4 & 5 & 450 & 10 & & & 66 & 67 \\
\hline & & & & 5 & & 1 & & & & 6 \\
\hline & 1 & 1 & & & 138 & 2 & & 3 & 1 & 1 \\
\hline & 6 & 2 & & & 4 & & & 1 & & \\
\hline & 3 & & & 2 & 3 & 1 & & & & \\
\hline \multirow{5}{*}{$\begin{array}{l}\text { AMERICAN ROBIN } \\
\text { BOHEMIAN WAXWING } \\
\text { CEDAR WAXWING } \\
\text { NORTHERN SHRIKE } \\
\text { EUROPEAN STARLING }\end{array}$} & & & & & 4 & & & & & \\
\hline & 143 & 12 & & 1 & 362 & 102 & & 2 & 120 & \\
\hline & & & & & 129 & & & & 103 & \\
\hline & 1 & & & & & & & & & \\
\hline & & & & & 19 & & & & & + \\
\hline \multirow{5}{*}{$\begin{array}{l}\text { DARK-EYED JUNCO (S- } \\
\text { SNOW BUNTING } \\
\text { PINE GROSBEAK } \\
\text { PURPLE FINCH } \\
\text { HOUSE FINCH }\end{array}$} & & & & & 5 & & & & 2 & \\
\hline & & & 211 & 1 & 36 & 67 & & 35 & 80 & 11 \\
\hline & 2 & 10 & & 8 & 62 & 6 & & 1 & 26 & 36 \\
\hline & & & & & & & & & & \\
\hline & & & & & 40 & & & 8 & & \\
\hline \multirow{5}{*}{$\begin{array}{l}\text { COMMON REDPOLL } \\
\text { HOARY REDPOLL } \\
\text { PINE SISKIN } \\
\text { EVENING GROSBEAK } \\
\text { HOUSE SPARROW }\end{array}$} & 40 & 28 & & 25 & 117 & 2 & & & 25 & 145 \\
\hline & & 4 & & & & & & & & \\
\hline & & & & & 6 & & & & & \\
\hline & 8 & & & 13 & & & & & & 239 \\
\hline & 23 & 48 & 82 & 33 & 2934 & 305 & 58 & 300 & 98 & \\
\hline \multirow{5}{*}{$\begin{array}{l}\text { TOTAL INDIVIDUALS COUNT DAY } \\
\text { NO. SPECIES COUNT DAY } \\
\text { NO. SPECIES COUNT PERIOD } \\
\text { NO. INDIVIDUALS IN TABLES } 4 \text { \& } 5 \\
\text { NO. SPECIES IN TABLES } 4 \& 5\end{array}$} & 454 & 167 & 469 & 153 & 8387 & 592 & 85 & 393 & 762 & 815 \\
\hline & 24 & 14 & 16 & 18 & 41 & 16 & 4 & 18 & 32 & 17 \\
\hline & 25 & 14 & 18 & 18 & 45 & 17 & 4 & 18 & 32 & 21 \\
\hline & 3 & 0 & 5 & 0 & 76 & 0 & 0 & 1 & 15 & 1 \\
\hline & 3 & 0 & 5 & 0 & 12 & 0 & 0 & 1 & 5 & 3 \\
\hline
\end{tabular}


Table 3-10. SPECIES RECORDED FROM MORE THAN NINE LOCALITIES $(+=$ species seen during the count period but not on count day)

\begin{tabular}{|c|c|c|c|c|c|c|c|c|c|c|}
\hline 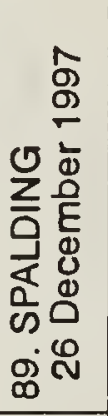 & 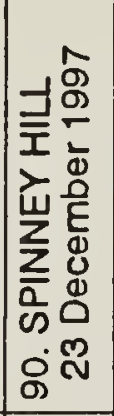 & 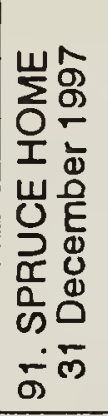 & 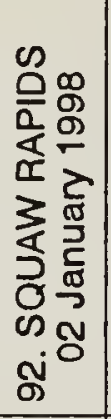 & 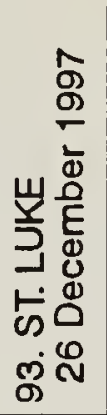 & 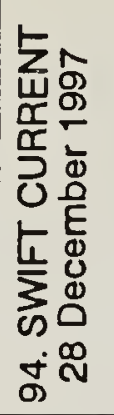 & 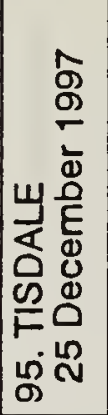 & 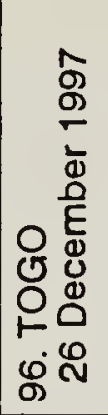 & 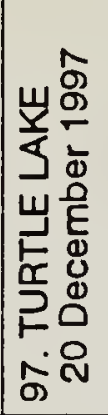 & 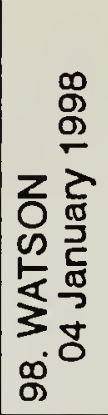 & 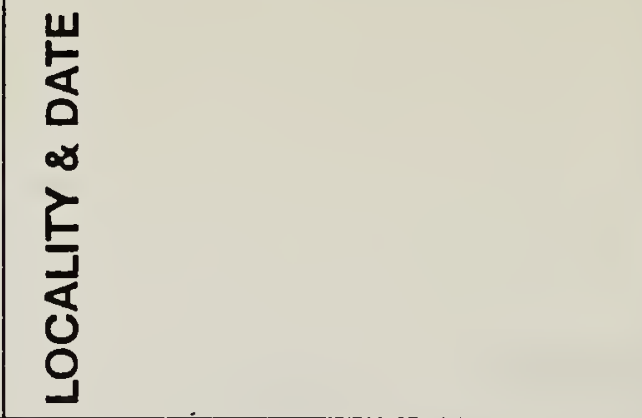 \\
\hline & & & 89 & & & & & & & \multirow{5}{*}{$\begin{array}{l}\text { CANADA GOOSE } \\
\text { MALLARD } \\
\text { COMMON GOLDENEYE } \\
\text { BALD EAGLE } \\
\text { NORTHERN GOSHAWK }\end{array}$} \\
\hline & & & 27 & & 2 & & & & & \\
\hline & & & 188 & & & & & & & \\
\hline & & & 75 & & & & & & & \\
\hline & & & 1 & & & & & & & \\
\hline & & & 1 & & & & & & & \multirow{5}{*}{$\begin{array}{l}\text { ROUGH-LEGGED HAWK } \\
\text { GOLDEN EAGLE } \\
\text { MERLIN } \\
\text { GRAY PARTRIDGE } \\
\text { RING-NECKED PHEASANT }\end{array}$} \\
\hline & & & & & & & 1 & & & \\
\hline & & & & & 3 & & & & & \\
\hline & & & & & 10 & & & & & \\
\hline & & & & & 1 & & & & & \\
\hline 2 & & & 4 & & & & & 6 & & \multirow{5}{*}{$\begin{array}{l}\text { RUFFED GROUSE } \\
\text { SHARP-TAILED GROUSE } \\
\text { ROCK DOVE } \\
\text { GREAT HORNED OWL } \\
\text { SNOWY OWL }\end{array}$} \\
\hline & & & 41 & 1 & & & & & & \\
\hline 3 & & & & 35 & 106 & & & 3 & 7 & \\
\hline & 1 & & 1 & 1 & & & & & & \\
\hline & 1 & & & & 1 & & & & & \\
\hline & 2 & 2 & 2 & 1 & 1 & & 2 & 6 & & \multirow{5}{*}{$\begin{array}{l}\text { DOWNY WOODPECKER } \\
\text { HAIRY WOODPECKER } \\
\text { BLACK-BACKED WOODPECKER } \\
\text { NORTHERN FLICKER (Y-S) } \\
\text { PILEATED WOODPECKER }\end{array}$} \\
\hline & 5 & 2 & 4 & & 1 & 1 & 2 & 8 & & \\
\hline & & & 1 & & & & & 1 & & \\
\hline & & & & & 5 & & & & & \\
\hline & & & 2 & & & & 1 & & & \\
\hline & & & & & 12 & & & & & \multirow{5}{*}{$\begin{array}{l}\text { HORNED LARK } \\
\text { GRAY JAY } \\
\text { BLUE JAY } \\
\text { BLACK-BILLED MAGPIE } \\
\text { COMMON RAVEN }\end{array}$} \\
\hline & & 1 & 11 & & & & & 7 & & \\
\hline & 8 & 1 & 7 & 1 & 1 & & & 39 & & \\
\hline 3 & 64 & 1 & 16 & 11 & 60 & 10 & 2 & 45 & 1 & \\
\hline 15 & 6 & 15 & 98 & + & & & 3 & 64 & 1 & \\
\hline 3 & 33 & 12 & 30 & 10 & + & 2 & 14 & 56 & 6 & \multirow{5}{*}{$\begin{array}{l}\text { BLACK-CAPPED CHICKADEE } \\
\text { BOREAL CHICKADEE } \\
\text { RED-BREASTED NUTHATCH } \\
\text { WHITE-BREASTED NUTHATCH } \\
\text { GOLDEN-CROWNED KINGLET }\end{array}$} \\
\hline & & & 7 & & & & & 16 & & \\
\hline & + & & & & 74 & & & 7 & & \\
\hline & & & 2 & & 2 & & 1 & 12 & & \\
\hline & 1 & & & & + & & & & & \\
\hline & & & & & + & & & & & \multirow{5}{*}{$\begin{array}{l}\text { AMERICAN ROBIN } \\
\text { BOHEMIAN WAXWING } \\
\text { CEDAR WAXWING } \\
\text { NORTHERN SHRIKE } \\
\text { EUROPEAN STARLING }\end{array}$} \\
\hline 40 & & & & 50 & 1 & 30 & & & + & \\
\hline & & & & & & & & & & \\
\hline & 1 & & & & & & 1 & & & \\
\hline & & & & & & & & & & \\
\hline & & & & & 4 & & & & & \multirow{5}{*}{$\begin{array}{l}\text { DARK-EYED JUNCO (S-C) } \\
\text { SNOW BUNTING } \\
\text { PINE GROSBEAK } \\
\text { PURPLE FINCH } \\
\text { HOUSE FINCH }\end{array}$} \\
\hline & & & 2 & & & & & & & \\
\hline . & 35 & 13 & 4 & 9 & 10 & & 10 & 121 & 6 & \\
\hline & & & & & 19 & & & & & \\
\hline & & & & & 1 & & & & & \\
\hline & 11 & 22 & 166 & 20 & & & 35 & 56 & & \multirow{5}{*}{$\begin{array}{l}\text { COMMON REDPOLL } \\
\text { HOARY REDPOLL } \\
\text { PINE SISKIN } \\
\text { EVENING GROSBEAK } \\
\text { HOUSE SPARROW }\end{array}$} \\
\hline & & & 8 & & & & 6 & 2 & & \\
\hline & & & & & 2 & & & 5 & & \\
\hline & 25 & 9 & 23 & & & & 13 & 27 & & \\
\hline 60 & 4 & & 6 & 26 & 1377 & 15 & & 30 & 16 & \\
\hline 126 & 197 & 78 & 831 & 166 & 1694 & 58 & 91 & 512 & 38 & \multirow{5}{*}{$\begin{array}{l}\text { TOTAL INDIVIDUALS COUNT DAY } \\
\text { NO. SPECIES COUNT DAY } \\
\text { NO. SPECIES COUNT PERIOD } \\
\text { NO. INDIVIDUALS IN TABLES } 4 \text { \& } 5 \\
\text { NO. SPECIES IN TABLES } 4 \text { \& } 5\end{array}$} \\
\hline 7 & 14 & 10 & 31 & 12 & 22 & 5 & 13 & 20 & 7 & \\
\hline 7 & 15 & 10 & 31 & 13 & 29 & 5 & 13 & 20 & 8 & \\
\hline 0 & 0 & 0 & 15 & 1 & 1 & 0 & 0 & 1 & 1 & \\
\hline 0 & 0 & 0 & 5 & 1 & 5 & 0 & 0 & 1 & 1 & \\
\hline
\end{tabular}


Table 3-11. SPECIES RECORDED FROM MORE THAN NINE LOCALITIES $(t=$ species seen during the count period but not on count day)

\section{SPECIES}

\section{CANADA GOOSE} MALLARD

COMMON GOLDENEYE

BALD EAGLE

NORTHERN GOSHAWK

ROUGH-LEGGED HAWK

GOLDEN EAGLE

MERLIN

GRAY PARTRIDGE

RING-NECKED PHEASANT

RUFFED GROUSE

SHARP-TAILED GROUSE

ROCK DOVE

GREAT HORNED OWL

SNOWY OWL

DOWNY WOODPECKER

HAIRY WOODPECKER

BLACK-BACKED WOODPECKER

NORTHERN FLICKER (Y-S)

PILEATED WOODPECKER

HORNED LARK

GRAY JAY

BLUE JAY

BLACK-BILLED MAGPIE

COMMON RAVEN

BLACK-CAPPED CHICKADEE

BOREAL CHICKADEE

RED-BREASTED NUTHATCH

WHITE-BREASTED NUTHATCH

GOLDEN-CROWNED KINGLET

AMERICAN ROBIN

BOHEMIAN WAXWING

CEDAR WAXWING

NORTHERN SHRIKE

EUROPEAN STARLING

DARK-EYED JUNCO (S-C)

SNOW BUNTING

PINE GROSBEAK

PURPLE FINCH

HOUSE FINCH

COMMON REDPOLL

HOARY REDPOLL

PINE SISKIN

EVENING GROSBEAK

HOUSE SPARROW

TOTAL INDIVIDUALS COUNT DAY

NO. SPECIES COUNT DAY

NO. SPECIES COUNT PERIOD

NO. INDIVIDUALS IN TABLES $4 \& 5$

NO. SPECIES IN TABLES 4 \& 5
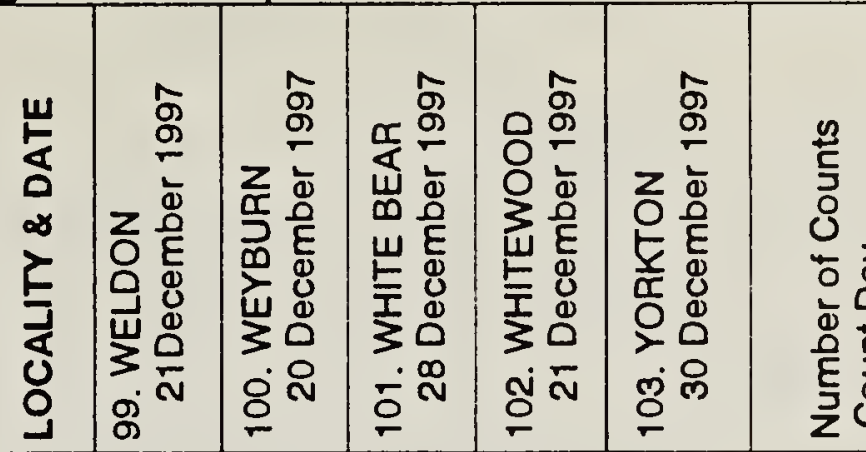

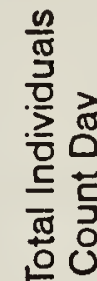

56832 27895

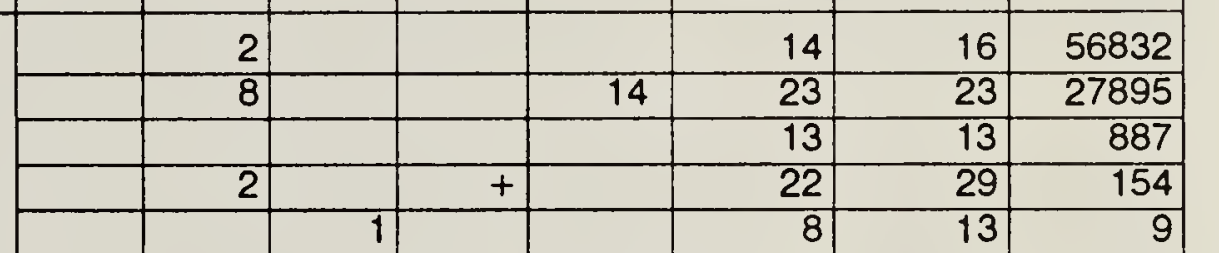

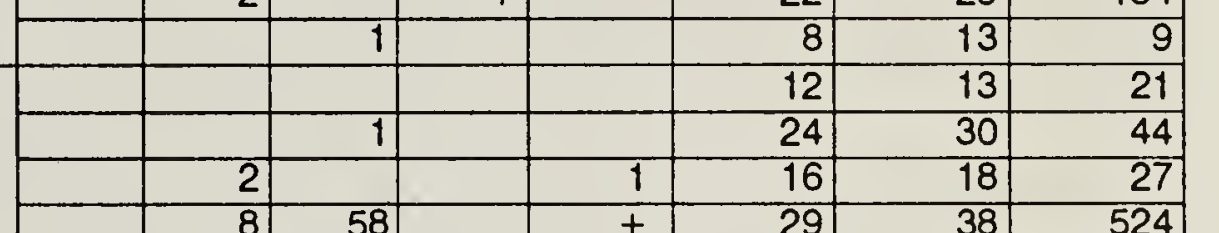

\begin{tabular}{|r|r|r|r|r|r|r|r|}
\hline & 8 & 58 & & + & 29 & 38 & 524 \\
\hline & & & & & 14 & 15 & 57 \\
\hline & & & 4 & 2 & 43 & 48 & 158 \\
\hline & 9 & 15 & 24 & & 40 & 45 & 998 \\
\hline
\end{tabular}

\begin{tabular}{|r|r|r|r|r|r|r|r|}
\hline & 9 & 15 & 24 & & 40 & 45 & 998 \\
\hline & 125 & 262 & 98 & 261 & 62 & 64 & 7568 \\
\hline & 4 & + & 3 & 1 & 53 & 63 & 127 \\
\hline & 2 & + & 2 & & 41 & 48 & 127 \\
\hline 2 & 5 & & 10 & 3 & 69 & 73 & 278 \\
\hline 2 & 1 & & 13 & 3 & 69 & 71 & 287 \\
\hline
\end{tabular}

\begin{tabular}{|c|c|c|c|c|c|c|c|}
\hline & & & & & 8 & 10 & 13 \\
\hline & 3 & & & 1 & 12 & 16 & 61 \\
\hline & & & & & 11 & 16 & 16 \\
\hline & & & + & & 18 & 22 & 416 \\
\hline
\end{tabular}

\begin{tabular}{|c|c|c|c|c|c|c|c|}
\hline & & & + & & 18 & 22 & 416 \\
\hline & & & & & 28 & 31 & 131 \\
\hline 2 & & & 3 & 5 & 71 & 75 & 703 \\
\hline 1 & 86 & 108 & 92 & 46 & 96 & 96 & 4985 \\
\hline 2 & & & 10 & 62 & 65 & 67 & 2207 \\
\hline 6 & 6 & & 89 & 60 & 87 & 89 & 3450 \\
\hline
\end{tabular}

\begin{tabular}{|r|r|r|r|r|r|r|r|}
\hline 6 & 6 & & 89 & 60 & 87 & 89 & 3450 \\
\hline & & & & 1 & 26 & 26 & 166 \\
\hline & 4 & & 8 & 11 & 54 & 56 & 705 \\
\hline 2 & 3 & & 4 & .1 & 41 & 42 & 171 \\
\hline & 2 & & & & 12 & 13 & 78 \\
\hline & & & + & 1 & 13 & 20 & 43 \\
\hline
\end{tabular}

\begin{tabular}{|c|c|c|c|c|c|c|c|}
\hline & & & + & 1 & 13 & 20 & \\
\hline 37 & & & 17 & 167 & 47 & 50 & 12722 \\
\hline & 2 & & + & & 9 & 11 & 477 \\
\hline & & & & & 10 & 10 & 14 \\
\hline & 31 & 2 & 18 & 8 & 18 & 19 & 351 \\
\hline & 7 & & 1 & & 18 & 18 & 152 \\
\hline & & & + & & 45 & 53 & 4762 \\
\hline 10 & 3 & & 13 & 40 & 69 & 70 & 1274 \\
\hline & 7 & & & & 7 & 10 & 53 \\
\hline & & & 2 & & 10 & 11 & 139 \\
\hline & 20 & & 306 & 40 & 64 & 65 & 3052 \\
\hline & & & 3 & 4 & 12 & 12 & 39 \\
\hline & 4 & & 1 & & 17 & 20 & 428 \\
\hline 15 & & & & & 42 & 45 & 2478 \\
\hline & 517 & 140 & 1285 & 297 & 84 & 85 & 22290 \\
\hline
\end{tabular}




\section{Table 4. SPECIES SEEN ON NINE OR FEWER COUNTS}

\section{SPECIES}

COMMON LOON

HORNED GREBE

WESTERN GREBE

AMERICAN WHITE PELICAN

DOUBLE-CRESTED CORMORANT

TUNDRA SWAN

TRUMPETER SWAN

GR. WHITE-FRONTED GOOSE

SNOW GOOSE

SNOW GOOSE (Blue)

GREEN-WINGED TEAL

AMERICAN BLACK DUCK

NORTHERN PINTAIL

NORTHERN SHOVELER

GADWALL

AMERICAN WIGEON

CANVASBACK

REDHEAD

RING-NECKED DUCK

GREATER SCAUP

LESSER SCAUP

OLDSQUAW

BUFFLEHEAD

HOODED MERGANSER

RED-BREASTED MERGANSER

COMMON MERGANSER

RUDDY DUCK

TURKEY VULTURE

NORTHERN HARRIER

SHARP-SHINNED HAWK

COOPER'S HAWK

SWAINSON'S HAWK

RED-TAILED HAWK

HARLAN'S HAWK

AMERICAN KESTREL

GYRFALCON

PRAIRIE FALCON

SPRUCE GROUSE

WILLOW PTARMIGAN

SAGE GROUSE

WILD TURKEY

AMERICAN COOT

KILLDEER

COMMON SNIPE

HERRING GULL

MOURNING DOVE

WESTERN SCREECH-OWL

NORTHERN HAWK-OWL

GREAT GRAY OWL

SHORT-EARED OWL

BOREAL OWL

THREE-TOED WOODPECKER

N. FLICKER (RED-SHAFTED)

\section{LOCATION AND NUMBER}

Squaw Rapids, 1

Coronach, 1

Gardiner Dam, 1

Fort Qu'appelle, 1; Squaw Rapids, 4

Squaw Rapids, 1

Craven, 1

Round Lake (Qu'appelle Valley), 1

Coronach, 1

Coronach, 3; Gardiner Dam, 1

Fort Qu'appelle, +

Coronach, 2; Fort Qu'appelle, +; Govenlock, +, Saskatchewan

Landing Prov. Park, 1

Coronach, 1

Coronach, 2; Fife Lake, 1

Coronach, 1

Coronach, 1; Crooked Lake, 1; Gardiner Dam, 1; Grayson, 2; Round Lake (Qu'appelle Valley), 1; Saskatchewan Landing Prov. Park, 1

Coronach, 1; Estevan, 1; Qu'appelle Valley Dam, 1; Saskatchewan Landing Prov. Park, 1

Coronach, 1; Gardiner Dam, 2

Coronach, 1; Grayson, 2

Saskatoon, 1

Gardiner Dam, 5

Beauval, +; Coronach, 5; Estevan, 2; Fort Qu'appelle, +; Gardiner

Dam, 7; Govenlock, 1

Saskatoon, 1

Coronach, 7; Fife Lake, 1

Coronach, 1; Fort Qu'appelle, +

Coronach, 2; Gardiner Dam, 3

Coronach, 7; Craven, 1; Fort Qu'appelle, + ; Gardiner Dam, 103;

Saskatoon, 2; Squaw Rapids, 1; Yorkton, 1

Kinloch, 1

Fort Walsh, 1; Pike Lake, 1

Saskatoon, +

Skull Creek, 1

Eastend - Consul, 1

Cypress Hills Prov. Park, 1; Fort Qu'appelle, +; Govenlock, 2;

Weyburn, 1

Govenlock, 1

Govenlock, 1; Whitewood, 1

Brightwater Reservoir, 1; Indian Head, 1; Love 1; Qu'appelle Valley

Dam, 1; Saskatchewan Landing Prov. Park, +

Govenlock, 2; Grasslands Nat. Park, 1; Rockglen - Borderland, +;

Skull Creek, 1; Swift Current. +; White Bear, +

Love 1

Nipawin; +; Snowden, +

Govenlock, 37

Broadview, 8; Estevan, 3; Estuary, 9; Fort Walsh, 1

Coronach, 51

Crooked Lake, +; Grayson, 1

Fort Walsh, 1; Saskatchewan Landing Prov. Park, 2

Gardiner Dam, 4

Govenlock, 1; Kinloch, +; Skull Creek, 1; St. Luke, 1

Leader South, 1

Prince Albert, 2; Snowden, +

Snowden, 1

Indian Head, 1; Regina, 2; Rockglen - Borderland, +

Anglin Lake, 1

Armit, 1; Duck Lake, 3; Fort Walsh, 1; Good Spirit L., 1; Horseshoe

Bend, 1; Kilwinning, 1; Prince Albert Nat. Park, 3

Regina, 1 
Table 4. SPECIES SEEN ON NINE OR FEWER COUNTS (continued)

\begin{tabular}{|c|c|}
\hline SPECIES & LOCATION AND NUMBER \\
\hline AMERICAN CROW & $\begin{array}{l}\text { Brightwater Reservoir, } 1 \text {; Fort Walsh, } 1 \text {; Kenosee Lake, } 1 \text {; Moose } \\
\text { Mountain, 1; Nipawin; } 2 \text {; Regina, } 4 \text {; Saskatoon, } 8 \text {; Weyburn, } 1 \text {; } \\
\text { Whitewood, }\end{array}$ \\
\hline MOUNTAIN CHICKADEE & Cypress Hills Prov. Park, 3 \\
\hline BROWN CREEPER & Fort Walsh, 4; Regina, 5 ; Swift Current. + \\
\hline TOWNSEND'S SOLITAIRE & $\begin{array}{l}\text { Fort Walsh, 1; Indian Head, 1; Kenosee Lake, 1; Moose Jaw, 1; } \\
\text { Regina, } 1\end{array}$ \\
\hline HERMIT THRUSH & MacDowall, + \\
\hline VARIED THRUSH & Prince Albert, +; Swift Current. + \\
\hline ORANGE-CROWNED WARBLER & Saskatoon, + \\
\hline YELLOW-RUMPED W. (MRYTLE) & Biggar, 1; Regina, 1 \\
\hline NORTHERN CARDINAL & Kamsack, 2 \\
\hline AMERICAN TREE SPARROW & Fort Walsh, 8; Govenlock, 1; Skull Creek, 9 \\
\hline SAVANNAH SPARROW & Gardiner Dam, 2; Rockglen - Borderland, 1 \\
\hline FOX SPARROW & Saskatoon, 1; Turtle Lake, 1 \\
\hline SONG SPARROW & Birch Hills, 1; Fort Walsh, 1 ; Kenaston, 1 \\
\hline LINCOLN'S SPARROW & Raymore, 1 \\
\hline WHITE-THROATED SPARROW & $\begin{array}{l}\text { Fort Qu'appelle, +; Kinloch, 1; Livelong, 1; Moose Jaw, +; } \\
\text { Saskatoon, 2; Swift Current. } 1\end{array}$ \\
\hline WHITE-CROWNED SPARROW & Fort Walsh, 1 \\
\hline HARRIS' SPARROW & Govenlock, 1; Round Lake (Qu'appelle Valley), 1 \\
\hline DARK-EYED JUNCO (OREGON) & Fort Walsh, 3 \\
\hline LAPLAND LONGSPUR & $\begin{array}{l}\text { Coronach, } 7 \text {; Fife Lake, 1; Fort Qu'appelle, 15; Govenlock, 3; } \\
\text { Livelong, 1; Moose Jaw, 1; Rockglen - Borderland, + }\end{array}$ \\
\hline RED-WINGED BLACKBIRD & Fort Qu'appelle, +; Fort Walsh, 2; Pike Lake, 1 \\
\hline RUSTY BLACKBIRD & Fort Walsh, 3; Govenlock,+ ; Kinloch, + ; Skull Creek, 3 \\
\hline BREWER'S BLACKBIRD & Biggar, 1; Govenlock , ; Pike Lake, 3; Preeceville, 1 ; Weyburn, 1 \\
\hline COMMON GRACKLE & $\begin{array}{l}\text { Gardiner Dam, 1; Horseshoe Bend, 1: Saskatoon, 1; Watson, 1; } \\
\text { Weyburn, } 1\end{array}$ \\
\hline GRAY-CROWNED ROSY FINCH & Cypress Hills Prov Park 2 ; Saskatoon, 1 ; Weldon, 1 \\
\hline RED CROSSBILL & Cypress Hills Prov. Park, 15; Fort Walsh, 149; Saskatoon, 9 \\
\hline WHITE-WINGED CROSSBILL & $\begin{array}{l}\text { Armit, 1; Fort Walsh, 26; Kelvington, } 6 \text {; Kinloch, 9; Prince Albert, } 7 \text {; } \\
\text { Raymore, 2; Regina, 14; Saskatoon, 50; Squaw Rapids, 8; } \\
\text { Swift Current. + }\end{array}$ \\
\hline AMERICAN GOLDFINCH & Shaunavon, 1 ; Whitewood, + \\
\hline
\end{tabular}

Table5 . BIRDS NOT IDENTIFIED TO SPECIES

\begin{tabular}{|l|l|}
\hline SPECIES & LOCATION AND NUMBER \\
\hline BUTEO species & Regina, 1 \\
\hline GULL (White-headed) species & Qu'appelle Valley Dam, 1 \\
\hline WOODPECKER species & Clark's Crossing, 1; Regina, 2 \\
\hline CHICKADEE species & Armit, 1 \\
\hline WAXWING species & Clark's Crossing, 50 \\
\hline REDPOLL species & Assiniboia, 40; Gardiner Dam, 2; Weldon, 24 \\
\hline
\end{tabular}


Table 6. SUMMARY OF NEW OR TYING HIGH COUNTS ESTABLISHED DURING THE 1997 COUNTS (tying counts regular type; new records are in bold/italic)

\begin{tabular}{|c|c|c|c|c|}
\hline LOCATION & $\begin{array}{r}1997 \\
\text { COUNT } \\
\end{array}$ & SPECIES & $\begin{array}{l}\text { EVIOUS } \\
\text { HIGH }\end{array}$ & LOCATION AND YEAR \\
\hline $\begin{array}{l}\text { Squaw Rapids } \\
\text { Coronach } \\
\text { Gardiner Dam }\end{array}$ & 1 & $\begin{array}{l}\text { COMMON LOON } \\
\text { HORNED GREBE } \\
\text { WESTERN GREBE }\end{array}$ & \begin{tabular}{l|}
1 \\
\end{tabular} & $\begin{array}{l}6 \text { counts N. to Grand Centre - } \\
\text { Pierceland and Squaw Rapids } \\
\text { Regina }(59,60,61,77) \\
\text { Saskatoon }(83) \\
\text { Regina }(56,69), \text { Qu'appelle Dam } \\
(90) \text {, Gardiner Dam }(79,88) \\
\end{array}$ \\
\hline $\begin{array}{l}\text { Squaw Rapids } \\
\text { Squaw Rapids } \\
\text { Coronach } \\
\text { Fort Qu'appelle } \\
\text { Estevan }\end{array}$ & $\begin{array}{r}4 \\
1 \\
3 \\
1 \\
27800 \\
\end{array}$ & \begin{tabular}{|l|} 
AM. WHITE PELICAN \\
DOUBLE-CRESTED COR- \\
$\quad$ MORANT \\
SNOW GOOSE \\
BLUE GOOSE \\
CANADA GOOSE \\
\end{tabular} & $\begin{array}{r}1 \\
\text { New } \\
3140 \\
\end{array}$ & $\begin{array}{l}\text { Regina (59), Love (91) } \\
\text { Squaw Rapids (81), } \\
\text { Gardiner Dam (87) } \\
\text { Regina (74, 84), Saskatoon (79) } \\
\text { Regina (93) }\end{array}$ \\
\hline \begin{tabular}{|l|} 
Coronach \\
Saskatoon \\
Gardiner Dam \\
Saskatoon \\
Coronach \\
Gardiner Dam \\
\end{tabular} & $\begin{array}{r}17000 \\
1 \\
5 \\
1 \\
7 \\
3 \\
\end{array}$ & \begin{tabular}{|l|} 
MALLARD \\
RING-NECKED DUCK \\
GREATER SCAUP \\
OLDSQUAW \\
BUFFLEHEAD \\
RED-BREASTED MERG. \\
\end{tabular} & $\begin{array}{r}15150 \\
1 \\
1 \\
+ \\
6 \\
3 \\
\end{array}$ & \begin{tabular}{|l|} 
Coronach (94) \\
Crooked Lake (89) \\
Regina (74) \\
Fort Qu'appelle (68) \\
Estevan \\
Gardiner Dam (87) \\
\end{tabular} \\
\hline $\begin{array}{l}\text { Skull Creek } \\
\text { Govenlock } \\
\text { Govenlock, Whitewood } \\
\text { Armit, Kamsack } \\
\text { Coronach } \\
\text { Leader South }\end{array}$ & $\begin{array}{r}1 \\
1 \\
1 \\
20 \\
51 \\
1 \\
\end{array}$ & \begin{tabular}{|l|} 
COOPER'S HAWK \\
HARLAN'S HAWK \\
AMERICAN KESTREL \\
RUFFED GROUSE \\
AMERICAN COOT \\
WESTERN SCREECH OWL \\
\end{tabular} & $\begin{array}{r}1 \\
1 \\
1 \\
17 \\
23 \\
\text { New } \\
\end{array}$ & $\begin{array}{l}10 \text { counts N. to North Battleford } \\
\text { White Bear (95) } \\
10 \text { counts N. to Dalmeny } \\
\text { Battlefords (60) } \\
\text { Coronach (94) } \\
\end{array}$ \\
\hline $\begin{array}{l}\text { Regina } \\
\text { Anglin Lake } \\
\text { Cypress Hills P. P. } \\
\text { MacDowall } \\
\text { Saskatoon } \\
\text { Biggar, Regina } \\
\end{array}$ & $\begin{array}{r}36 \\
1 \\
3 \\
+ \\
+ \\
1\end{array}$ & \begin{tabular}{|l|} 
SNOWY OWL \\
BOREAL OWL \\
MOUNTAIN CHICKADEE \\
HERMIT THRUSH \\
ORANGE-CROWNED W. \\
YELLOW-RUMP. W. (MOVte) \\
\end{tabular} & $\begin{array}{r}25 \\
1 \\
1 \\
\text { New } \\
\text { New } \\
1 \\
\end{array}$ & $\begin{array}{l}\text { Regina (79) } \\
20 \text { counts S. to Indian Head } \\
5 \text { counts N. to Marsden } \\
\text { Saskatoon (54) }\end{array}$ \\
\hline $\begin{array}{l}\text { Kamsack } \\
\text { Gardiner Dam } \\
\text { Saskatoon, Turtie Lake } \\
\text { Raymore } \\
\text { Fort Walsh } \\
\text { Swift Current }\end{array}$ & \begin{tabular}{r|}
2 \\
2 \\
1 \\
1 \\
1 \\
19
\end{tabular} & \begin{tabular}{|l|} 
NORTHERN CARDINAL \\
SAVANNAH SPARROW \\
FOX SPARROW \\
LINCOLN'S SPARROW \\
WHIE-CROWNESPARPOW \\
PURPLE FINCH
\end{tabular} & \begin{tabular}{r|}
1 \\
1 \\
1 \\
1 \\
1 \\
18
\end{tabular} & $\begin{array}{l}\text { Craven (60), Saskatoon }(80,81) \\
\text { Saskatoon (91) } \\
6 \text { counts N. to Biggar and Saskatoon } \\
\text { Broadview (95), Gardiner Dam (96) } \\
7 \text { counts N. to Saskatoon } \\
\text { Kamsack (91) }\end{array}$ \\
\hline
\end{tabular}


Table 7. COMPARISON OF THE 1997 POPULATIONS TO 1996 AND AVERAGE PAST YEARS (1992 - 1996) BASED ON PARTY HOURS PER INDIVIDUAL BIRD SEEN

\begin{tabular}{|c|c|c|c|c|c|}
\hline SPECIES & 97 VS 96 & vs $5 y r$ & SPECIES & 97 VS 96 & vs $5 y r$ \\
\hline CANADA GOOSE & 29711.4 & 2582.1 & COMMON RAVEN & 18.6 & 20.5 \\
\hline & & & PPED CHICKADEE & -13.4 & -0.9 \\
\hline COMMON GOLDENEYE & 217.9 & -4.1 & SHICKADEE & 27.5 & 22.6 \\
\hline BALD EAGLE & 181.9 & 93.5 & ED-BREASTED NUTHATCH & 68.0 & 38.1 \\
\hline NORTHERN GOSHAWK & -64.8 & -4.2 & WHITE-BREASTED NUTHATCH & 9.8 & -1.8 \\
\hline GOLDEN EAGLE & 22.1 & -8.0 & BROWN CREEPER & 10.6 & 10.4 \\
\hline & 78.7 & 7. & VED KINGLET & 6610.1 & 15.2 \\
\hline FALCON & 14.7 & & & & -2.5 \\
\hline $\mathrm{GE}$ & -82.3 & -80 & WING & 242.7 & 109.1 \\
\hline PHEASANT & -74.2 & -9.1 & CEDAR WAXWING & 1.3 & -49.5 \\
\hline USE & -95.2 & -32.4 & & -45.3 & -59.2 \\
\hline & & & & -28.1 & -35.3 \\
\hline GROUSE & & & PARROW & 93.6 & -5.6 \\
\hline & & & & 18.9 & -33.3 \\
\hline RNED OWL & 40.1 & 14.1 & GSPUR & -64.0 & -99.2 \\
\hline YOWL & 45.7 & 24.0 & & -73.7 & -96.0 \\
\hline RED OWL & & & LACKB & & -115.9 \\
\hline DPECKER & -21.1 & -12 & & -83.9 & -107.2 \\
\hline ECKE & -21.6 & -21.4 & & -43.3 & -17.7 \\
\hline THREE-TOED WOODPECKER & -27.2 & 1.0 & BILL & -20.4 & 839.2 \\
\hline NORTHERN FLICKER & 249.8 & 93.8 & D CROSSBILL & -70.6 & -92.4 \\
\hline PILEATED WOODPECKER & 14.7 & & (1) & -2.4 & -33.9 \\
\hline HORNED LARK & -58.2 & -93.4 & EDPOLL & 59.8 & -53.8 \\
\hline & 34.2 & -0.6 & & 338.3 & 366.7 \\
\hline BLUE JAY & 10.6 & 24. & EVENING GROSBEAK & 66.5 & 22.7 \\
\hline BLACK-BILLED MAGPIE & 3.4 & 21.5 & HOUSE SPARROW & 37.9 & 14.3 \\
\hline
\end{tabular}

